
$\$$ Research Square
Preprints are preliminary reports that have not undergone peer review.
They should not be considered conclusive, used to inform clinical practice, or referenced by the media as validated information.

\title{
The landscape of tumor mutational burden and its clinical significance in patients with lung cancer: a Multi-omics study with a meta-analysis
}

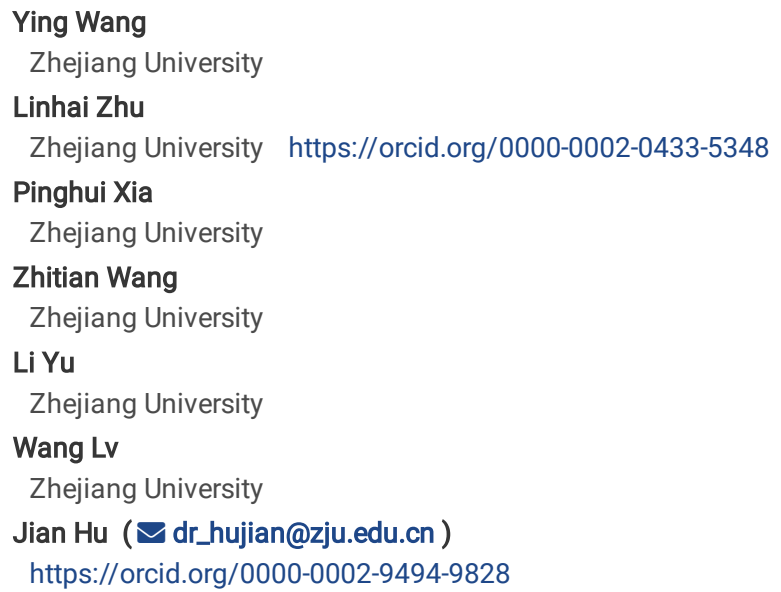




\section{Abstract}

Background: Current research on tumor mutational burden (TMB) has focused on tumor immunotherapy responsiveness, but the role of TMB in nonimmunotherapy patients is unclear. The purpose of this study is to explore the effect of TMB on lung cancer patients in order to clarify and expand the clinical significance of TMB in lung cancer.

Methods: We download mutation data of lung cancer cases from The Cancer Genome Atlas (TCGA) database to analyze TMB and its composition, and study the relationship between TMB and clinicopathological characteristics of lung cancer patients. We then systematically retrieved and analyzed studies on the relationship between TMB and survival outcomes. The hazard ratio (HR) and its $95 \%$ confidence interval (Cl) were used as an effective size to assess the survival outcomes. The subgroup analyses based on the pathological type, treatment method, TMB detection method and detection materials were also performed to explore the factors that might affect the interpretation of TMB results.

Results: TMB in lung squamous cell carcinoma is lower than those in lung adenocarcinoma. In lung adenocarcinoma, patients with EGFR mutation have lower TMB than patients with EGFR wild-type. The summary analysis found that TMB is a better prognostic factor in small cell lung cancer, and more evident in small cell lung cancer receiving immunotherapy. TMB is a neutral or poor prognostic indicator in non-small cell lung cancer, but a better prognostic factor in non-small cell lung cancer receiving immunotherapy. In patients with lung adenocarcinoma, including those with EGFR mutation and receiving EGFR-targeted therapies, high TMB means worse survival. TMB detected by blood specimens is inconsistent and unstable compared to TMB detected by tissue. The clinical significance of TMB from blood specimens needs further study on extensive sample data.

Conclusions: The pooled results indicated that TMB is a good prognostic factor in lung cancer patients receiving immunotherapy. But high TMB is connected with worse survival in non-small cell lung cancer without receiving immunotherapy, especially in lung adenocarcinoma. For lung adenocarcinoma patients with both EGFR mutation and high TMB, how to make a choice between EGFR-targeted therapy and immunotherapy is still a problem that requires further research.

\section{Background}

Lung cancer, including non-small cell lung cancer (NSCLC) and small cell lung cancer (SCLC), is the most common malignant tumor and the leading cause of tumor-related death in the world [1]. NSCLC, accounting for approximately $85 \%$ of all lung cancer incidences, is mainly composed of lung adenocarcinoma (LUAD), lung squamous cell carcinoma (LUSC), and lung large cell carcinoma [2]. SCLC, accounting for around 10-15\% of all lung cancers, is characterized by neuroendocrine and has a high degree of malignancy [3]. Different pathological types of lung cancer have different biological characteristics and various treatment options [4-6]. The clinical application of targeted therapy has led to a dramatic change in the treatment of patients with LUAD [7, 8]. Unfortunately, targeted therapy for oncogenic driver mutations in EGFR or ALK fusions that works on LUAD are generally ineffective against LUSC and SCLC [5, 9]. Immunotherapy based on immune checkpoint inhibitors (ICls) provides definite efficacy for patients with chemotherapy-resistant lung cancer, and is gradually moving towards first-line treatment for lung cancer [10]. But there are also some patients who have no response to ICI therapy, especially those containing oncogenic driver mutation [11].

With the development of next-generation sequencing technology, whole-exome sequencing (WES) and targeted next-generation sequencing (targeted NGS) have been used in clinical decision-making [12]. The changes in tumor driver genes such as EGFR, ALK, MET, BRAF, ROS1, HER2 can be detected based on NGS, and those results are used to guide the application of targeted drugs [13]. Tumor mutational burden (TMB), as a product of the era of NGS, is also gradually widely used in clinical practice [14]. Under this circumstance, how to precisely understand the meaning of TMB and use it to manage lung cancer is of great practical significance.

In addition to PD-L1 expression, microsatellite instability (MSI), and mismatch repair deficiency (MMR), TMB also has emerged as a promising biomarker for response to ICls therapy in clinical trials [15]. The hypothesis that tumors with more neoantigens may respond better to immunotherapy is based on the positive relationship between tumor-specific neoantigens and increased immunogenicity [16]. Most current researches focus on the relationship between TMB and the efficacy of immunotherapy in various cancers [17-19]. However, the role of TMB in lung cancer patients without receiving immunotherapy has not been fully elucidated. TMB is counted by the sum of non-synonymous mutations that also contain the oncogenic driver mutations, but the additional mutations may constitute potential resistance pathways to targeted therapies [11]. In order to expand the interpretation of the potential clinical significance of TMB, we analyzed the composition of TMB in lung cancer, and then reviewed the role of TMB in lung cancer patients with different characteristics.

\section{Methods}

\subsection{Multi-omics study}

\subsubsection{Data acquisition and preprocessing}

First, we download the somatic mutation data of patients with LUAD and LUSC respectively from "simple nucleotide variation" category in The Cancer Genome Atlas (TCGA) database (http://portal.gdc.cancer.gov/). The "Masked Somatic Mutation" data processed by VarScan2 software was used for further TMB analysis. Then, the transcriptome data with HTSeq-FPKM format of patients with LUAD and LUSC were download from "transcriptome profiling" in TCGA database. Moreover, the clinical information of LUAD and LUSC patients, including age, gender, TNM stages, and survival status were all obtained from the TCGA database. Next step, all transcriptome data and clinical data were extracted from the corresponding single files and merged. 


\subsubsection{The landscape of $T M B$ for each patient and survival analysis}

In this study, TMB was defined as the total number of variants, which include detected base deletions, insertions or substitutions. The "maftools" package running in R software is used to analyze the genomic mutation profiles and create visualizations using the "Masked Somatic Mutation" data [20]. A Perl script was used to extract the genomic mutations of patients with LUAD or LUSC, and calculate the TMB value for each patient. TMB values were then merged with corresponding clinical data and genetic mutation data, respectively. The LUAD or LUSC patients were divided into high TMB and low TMB groups by median TMB value. Kaplan-Meier analysis with log-rank test was used to evaluate the difference in overall survival (OS) between the two groups. In addition, we assessed the relationship between TMB and several clinicopathological characteristics.

\subsubsection{Statistical Analysis}

The Wilcoxon rank-sum test was applied for comparison of continuous variables, and the chi-square test was used for comparison of categorical variables. The Kaplan-Meier analysis with log-rank test was performed by the "survival" package of the R software.

All the statistical analyses were conducted in R software (version 3.6.0). All the tests were two-sided, and a P-value less than 0.05 was considered to be significant.

\subsection{Systematic Review And Meta-analysis}

\subsubsection{Literature search strategy}

The systematic review and meta-analysis were performed based on the Preferred Reporting Items for Systematic Reviews and Meta-analyses (PRISMA) statement [21]. The electronic databases, including PubMed, PubMed Central (PMC), EMBASE, and Ovid, were used to search related pieces of literature from inception to January 11, 2020. Search terms included the following: "lung cancer or lung carcinoma" and "TMB or tumor mutation burden" and "survival or prognosis". All references lists of identified articles were also manually searched for potentially relevant reports.

\subsubsection{Inclusion And Exclusion Criteria}

The eligibility criteria for included studies were as follow: 1) RCT or cohort studies to study the role of TMB as a biomarker for lung cancer prognosis, and 2) studies comparatively analyze the survival results of lung cancer patients with high TMB and those with low TMB. The exclusion criteria were as follow: 1) review articles without original data, 2) studies focused on the TMB detection technology, 3) studies without TMB-related survival data, and 4) studies in nonlung cancer. For multiple articles reporting a duplicate population, only the most complete or recent one was included. Two reviewers independently performed the selection process of eligible studies, and differences were resolved through discussion.

\subsubsection{Data Extraction And Quality Assessment}

Two reviewers independently collected the following data using a predesigned form: first author, publication time, study period, country, sample size (low- and high- TMB), age (low- and high- TMB), gender (low- and high- TMB), tumor histology (low- and high- TMB), tumor stage (low- and high- TMB), driver mutation, treatment, sample source, TMB detection method, cutoff value, follow-up time, and primary endpoint. Disputes in this process were handled through discussion. The hazard ratio (HR) and 95\% confidence intervals ( $\mathrm{Cl}$ ) of the high TMB group compared to the low TMB group for OS and progression-free survival (PFS) were primarily collected. Disease-free survival (DFS) was considered as PFS for further analysis due to the similar clinical significance of DFS and PFS. For studies in which the HR and $95 \% \mathrm{Cl}$ were not provided explicitly, we used Tierney's methods to extract survival data from the Kaplan-Meier curve or the original data [22]. If the above item could not be obtained in the original study, this item was marked as "not acquired (NA)". Two reviewers independently assessed the quality of included cohort studies based on the Newcastle-Ottawa Scale (NOS) [23]. The NOS evaluated the quality of a survey with a scale ranged from 0 to 9 . Studies with a score of 6 or high were deemed as high-quality studies.

\subsubsection{Statistical Analysis}

The HR and its $95 \% \mathrm{Cl}$ were used as effect size (ES) to assess the role of TMB in the survival of lung cancer patients. Heterogeneity across the studies was evaluated using I-squared statistics [24]. A random-effect model was utilized if $\mathrm{I}^{2}$ greater than 50\%; Otherwise, a fixed-effect model was chosen. To detect the different prognostic effects of TMB in different pathological types of lung cancer, various treatments, different methods of TMB detection and diverse sampling, we performed the subgroup analyses based on different scenarios. To confirm the robustness of the pooled results, sensitivity analyses by eliminating a single study at a time were adopted. Potential publication bias was assessed by Begg's funnel plot and Egger's test. The statistical analyses were conducted using the software STATA version 12.0 (Stata Inc, TX, USA). All the tests were two-sided, and a P value less than 0.05 was considered to be significant.

\section{Results}

\subsection{Multi-omics study}

Page $3 / 20$ 


\subsubsection{Genome-wide mutation spectrum in LUAD and LUSC}

The gene mutation data, transcriptome data, and clinical information of 585 LUAD patients and 504 LUSC patients were obtained from the TCGA cohorts. Of the 585 LUAD patients, 569 has simple nucleotide variation data, 515 has transcriptome data, and 522 the clinical data. Of the 504 LUSC cases, 497 had simple nucleotide variation data, 501 had transcriptome data, and 504 had clinical data. TMB values were extracted from 561 LUAD cases and 491 LUSC cases. We combined TMB values with clinical data and expression profile data, and cases with incomplete data were discarded. Genome-wide mutation profiling was visualized via "maftools" package. Overall mutation types in patients with LUAD and LUSC were shown in Figs. 1 and 2. In both types of lung cancer, missense mutations were the most common type of mutation (Fig. 1A and 2A). Compared with insertion and deletion, single-nuclear polymorphisms (SNPs) accounted for the vast majority (Fig. 1B and 2B), of which $\mathrm{C}>\mathrm{A}$ and $\mathrm{C}>\mathrm{T}$ were the most common types (Fig. $1 \mathrm{C}$ and $2 \mathrm{C}$ ). Waterfall plots revealed the top 80 mutant genes with the highest mutation frequency in patients with LUAD (Fig. 1D) and LUSC (Fig. 2D). Various colors in the waterfall chart represented different types of mutations. TP53 (47\%), TTN (41\%), MUC16 (40\%), RYR2 (34\%), CSMD3 (34\%), LRP1B (29\%), ZFHX4 (27\%), USH2A (27\%), KAS (25\%), and XIPR2 (22\%) were the top 10 mutated genes in LUAD. EGFR mutation frequency in LUAD is $12 \%$, ranking 53rd. The top ten mutations in LUSC were TP53 (77\%), TTN (68\%), CSMD3 (40\%), MUC16 (36\%), RYR2 (35\%), LRP1B (30\%), USH2A (30\%), SYNE1 (29\%), ZFHX4 (26\%), and KMT2D (22\%). The total number of mutated bases in each patient is shown in Fig. 3. The median TMB was 140 mutations in LUAD (Fig. 3A) and 182 mutations in LUSC (Fig. 3B).

\subsubsection{Relationship Between Tmb And Clinicopathological Characteristics}

The clinicopathological characteristics of LUAD and LUSC patients were summarized in Table 1. In the LUAD cohort, the age of the low TMB group was higher than that of the high TMB group $(P=0.003)$. There were more women in the low TMB group and more men in the high TMB group $(P=0.014)$. In the LUSC cohort, the age of the low TMB group was also higher than that of the high TMB group, but the statistical significance was not significant $(P=0.061)$. The proportion of stage I patients is higher in the low TMB group, and the ratio of stage III-IV patients in the high TMB group is higher ( $P=0.004)$. Kaplan-Meier analysis with log-rank test showed that TMB did not significantly affect OS in LUAD (Fig. 4A, $P=0.983$ ) and LUSC (Fig. 4B, $P=0.602$ ).

Table 1

Clinicopathological characteristics of LUAD and LUSC patients from TCGA cohort

\begin{tabular}{|c|c|c|c|c|c|c|}
\hline & \multicolumn{3}{|l|}{ LUAD } & \multicolumn{3}{|l|}{ LUSC } \\
\hline & Low-TMB & High-TMB & $P$ value & Low-TMB & High-TMB & $P$ value \\
\hline $\begin{array}{l}\text { Age, } \\
\text { year }\end{array}$ & $\begin{array}{l}\text { Median 67.0, } \\
\text { range }(41-86)\end{array}$ & $\begin{array}{l}\text { Median 64.5, } \\
\text { range (33-88) }\end{array}$ & 0.003 & $\begin{array}{l}\text { Median 69.0, } \\
\text { range (39-90) }\end{array}$ & $\begin{array}{l}\text { Median 67.0, } \\
\text { range (40-83) }\end{array}$ & 0.061 \\
\hline \multicolumn{7}{|l|}{ Gender } \\
\hline Female & $150(59.1 \%)$ & $123(48.2 \%)$ & 0.014 & $58(23.9 \%)$ & 69 (27.8\%) & 0.317 \\
\hline Male & 104 (40.9\%) & 132 (51.8\%) & & $185(76.1 \%)$ & $179(72.2 \%)$ & \\
\hline \multicolumn{7}{|l|}{ Stage } \\
\hline I & 137 (53.9\%) & 139 (54.5\%) & 0.998 & 131 (53.9\%) & 108 (43.5\%) & 0.004 \\
\hline II & $63(24.8 \%)$ & $60(23.5 \%)$ & & 81 (33.3\%) & 78 (31.5\%) & \\
\hline III & $41(16.1 \%)$ & $42(16.5 \%)$ & & 29 (11.9\%) & $56(22.6 \%)$ & \\
\hline IV & 12 (4.7\%) & $13(5.1 \%)$ & & $1(0.4 \%)$ & $6(2.4 \%)$ & \\
\hline Unknown & $1(0.4 \%)$ & $1(0.4 \%)$ & & $1(0.4 \%)$ & $0(0 \%)$ & \\
\hline
\end{tabular}

To explore the possible efficacy of ICl immunotherapy in EGFR mutation-driven lung cancer patients, we examined the relationship between EGFR mutation status and two predictive indicators commonly used in immunotherapy, TMB value and PD-L1 expression. Patients with EGFR mutations in LUAD had significantly lower TMB values than those with EGFR wild type (Fig. 1D, the red line indicates the main population of EGFR mutations; Fig. 5A, $P=5.225 \mathrm{e}-07$ ). However, there was no significant difference in TMB values between the EGFR mutation group and wild type (WT) group in LUSC (Fig. 5B, $P=0.678$ ). There was also no obvious correlation between EGFR mutation and PD-L1 mRNA expression in LUAD (Fig. 6A, P=0.299) and LUSC (Fig. 6B, P=0.779).

\subsection{Systematic Review And Meta-analysis}

\subsubsection{Search results and study characteristics}

The literature selection process was shown in Fig. 7. After removing duplicates, a total of 24229 records were retrieved by the search strategy above. By viewing titles and abstracts, 24115 irrelevant articles were excluded. The remaining 114 articles were read in full-text. Finally, 30 articles were included in this analysis. 
Thirty eligible articles included 37 independent cohort studies. The main characteristics of the 37 studies were shown in Table 2 . Thirty-one studies report the role of TMB in NSCLC and six studies indicate the role in SCLC. Based on the NOS score, these 37 studies have a quality of 6 to 9 , with an average score of 7.2 . 
Table 2

Characteristics of studies included in the $\mathrm{r}$

\begin{tabular}{|c|c|c|c|c|c|c|c|c|c|c|c|c|c|}
\hline \multirow[b]{2}{*}{ Author } & \multirow[b]{2}{*}{ Year } & \multirow[b]{2}{*}{ Period } & \multirow[b]{2}{*}{ Country } & \multicolumn{2}{|c|}{ Sample size } & \multicolumn{2}{|l|}{ Age } & \multicolumn{2}{|c|}{ Gender } & \multicolumn{2}{|c|}{ Tumor histology } & \multicolumn{2}{|l|}{ Stage } \\
\hline & & & & $\begin{array}{l}\text { TMB } \\
\text { low }\end{array}$ & $\begin{array}{l}\text { TMB } \\
\text { high }\end{array}$ & $\begin{array}{l}\text { TMB } \\
\text { low }\end{array}$ & $\begin{array}{l}\text { TMB } \\
\text { high }\end{array}$ & $\begin{array}{l}\text { TMB } \\
\text { low }\end{array}$ & $\begin{array}{l}\text { TMB } \\
\text { high }\end{array}$ & TMB low & $\begin{array}{l}\text { TMB } \\
\text { high }\end{array}$ & TMB low & TMB high \\
\hline $\begin{array}{l}\text { Alborelli I } \\
\text { [16] }\end{array}$ & 2020 & $\begin{array}{l}2013- \\
2018\end{array}$ & Switzerland & 51 & 25 & $\begin{array}{l}\text { Median } \\
65, \\
\text { range } \\
(49- \\
79)\end{array}$ & $\begin{array}{l}\text { Median } \\
67, \\
\text { range } \\
(31- \\
90)\end{array}$ & $\begin{array}{l}M \\
30, F \\
21\end{array}$ & $\begin{array}{l}M \\
16, F \\
8\end{array}$ & $\begin{array}{l}\text { LUAD 47, } \\
\text { LUSC } 4\end{array}$ & $\begin{array}{l}\text { LUAD 23, } \\
\text { LUSC } 2\end{array}$ & $\begin{array}{l}\text { I-III 17, IV } \\
32\end{array}$ & $\begin{array}{l}\mathrm{I}-\mathrm{III} 8, \mathrm{IV} \\
17\end{array}$ \\
\hline $\begin{array}{l}\text { Ricciuti B } \\
\text { [45] }\end{array}$ & 2019 & $\begin{array}{l}2014- \\
2018\end{array}$ & USA & 26 & 26 & $\begin{array}{l}\text { Median } \\
67.5 \text {, } \\
\text { range } \\
(43- \\
83)\end{array}$ & $\begin{array}{l}\text { Median } \\
63.5, \\
\text { range } \\
(47- \\
84)\end{array}$ & $\begin{array}{l}M \\
15, F \\
11\end{array}$ & $\begin{array}{l}M \\
10, F \\
16\end{array}$ & SCLC & SCLC & $\begin{array}{l}\text { Extensive } \\
19, \\
\text { Limited } 7\end{array}$ & $\begin{array}{l}\text { Extensive } \\
15, \\
\text { Limited } 11\end{array}$ \\
\hline $\begin{array}{l}\text { Ricciuti B } \\
\text { [45] }\end{array}$ & 2019 & $\begin{array}{l}2014- \\
2018\end{array}$ & USA & 38 & 23 & NA & NA & NA & NA & SCLC & SCLC & Extensive & Extensive \\
\hline $\begin{array}{l}\text { Samstein } \\
\text { RM [30] }\end{array}$ & 2019 & $\begin{array}{l}2013- \\
2017\end{array}$ & USA & 280 & 70 & $\begin{array}{l}\text { range } \\
31-90\end{array}$ & $\begin{array}{l}\text { range } \\
31-90\end{array}$ & $\begin{array}{l}M \\
136 \\
F \\
144\end{array}$ & $\begin{array}{l}M \\
34, F \\
36\end{array}$ & NSCLC & NSCLC & Advanced & Advanced \\
\hline $\begin{array}{l}\text { Samstein } \\
\text { RM [30] }\end{array}$ & 2019 & $\begin{array}{l}2013- \\
2017\end{array}$ & USA & 498 & 125 & NA & NA & NA & NA & NSCLC & NSCLC & Advanced & Advanced \\
\hline Offin M [11] & 2019 & $\begin{array}{l}2010- \\
2017\end{array}$ & USA & 102 & 51 & NA & NA & $\begin{array}{l}M \\
30, F \\
72\end{array}$ & $\begin{array}{l}M \\
23, F \\
28\end{array}$ & NSCLC & NSCLC & Metastatic & Metastatic \\
\hline Jiao XD [25] & 2019 & $\begin{array}{l}2009- \\
2014\end{array}$ & USA & 111 & 178 & NA & NA & NA & NA & LUAD & LUAD & Advanced & Advanced \\
\hline Zhou M [46] & 2019 & $\begin{array}{l}2014- \\
2017\end{array}$ & China & 26 & 27 & $₫ 6025, \geq 6$ & 028 & M 49, & & SCLC & SCLC & Limited 24, E & xtensive 29 \\
\hline Chen Y [26] & 2019 & $\begin{array}{l}2009- \\
2016\end{array}$ & China & 118 & 18 & $\begin{array}{l}\otimes 6055, \\
\geq 60 \\
63\end{array}$ & $\begin{array}{l}8609 \\
\geq 609\end{array}$ & $\begin{array}{l}M \\
71, F \\
47\end{array}$ & $\begin{array}{l}M \\
15, F \\
3\end{array}$ & LUAD & LUAD & $\begin{array}{l}\text { IIIB 46, IV } \\
72\end{array}$ & $\begin{array}{l}\text { IIIB 8, IV } \\
10\end{array}$ \\
\hline Chen Y [26] & 2019 & $\begin{array}{l}2009- \\
2016\end{array}$ & China & 37 & 14 & 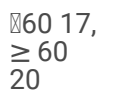 & $\begin{array}{l}8607 \\
\geq 607\end{array}$ & $\begin{array}{l}M \\
28, F \\
9\end{array}$ & $\begin{array}{l}M \\
12, F \\
2\end{array}$ & LUSC & LUSC & $\begin{array}{l}\text { IIIB 14, IV } \\
23\end{array}$ & IIIB 7, IV 7 \\
\hline Heeke S [47] & 2019 & $\begin{array}{l}2016- \\
2018\end{array}$ & France & 21 & 15 & $\begin{array}{l}\text { Median } \\
59.2, \\
\text { range } \\
(44.5- \\
82.2)\end{array}$ & $\begin{array}{l}\text { Median } \\
60.2, \\
\text { range } \\
(37.6- \\
83.1)\end{array}$ & $\begin{array}{l}M \\
16, F \\
5\end{array}$ & $\begin{array}{l}M \\
15, F \\
0\end{array}$ & $\begin{array}{l}\text { LUAD 10, } \\
\text { LUSC 9, } \\
\text { LCNC 1, } \\
\text { PSC } 1\end{array}$ & $\begin{array}{l}\text { LUAD 11, } \\
\text { LUSC } 3 \\
\text { PSC } 1\end{array}$ & $\begin{array}{l}\text { | 0, || 3, I|| } \\
9, \text { IV } 9\end{array}$ & $\begin{array}{l}|2,||| 2,|| \mid \\
2, \text { IV } 9\end{array}$ \\
\hline Jiang T [48] & 2019 & $\begin{array}{l}2012- \\
2015\end{array}$ & China & 94 & 95 & 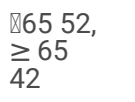 & $\begin{array}{l}\otimes 6553 \\
\geq 6542\end{array}$ & $\begin{array}{l}M \\
88, F \\
6\end{array}$ & $\begin{array}{l}M \\
91, F \\
4\end{array}$ & LUSC & LUSC & $\begin{array}{l}\text { | 47, || 29, } \\
\text { III } 18\end{array}$ & $\begin{array}{l}\text { | } 45, \text { II } 25, \\
\text { III } 25\end{array}$ \\
\hline Ready N [49] & 2019 & 2016 & $\begin{array}{l}\text { USA and } \\
\text { Canada }\end{array}$ & 50 & 48 & $65.5(42-\varepsilon$ & 85) & M 54, & 44 & $\begin{array}{l}\text { LUSC } 34, n \\
64\end{array}$ & n-LUSC & IIIB 20, IV 78 & \\
\hline Yu H [50] & 2019 & NA & USA & 78 & 77 & $\begin{array}{l}\text { Median } \\
71.73, \\
\text { range } \\
(43- \\
86.18)\end{array}$ & $\begin{array}{l}\text { Median } \\
70.77, \\
\text { range } \\
(44.81- \\
92.22)\end{array}$ & $\begin{array}{l}M \\
53, F \\
25\end{array}$ & $\begin{array}{l}\mathrm{M} \\
102 \\
\mathrm{~F} 28\end{array}$ & LUSC & LUSC & $\mathrm{I}-\mathrm{II}$ & I-II \\
\hline Wang C [51] & 2019 & NA & China & 68 & 33 & $\begin{array}{l}\text { Median } 59 \\
\text { range (25- }\end{array}$ & $\begin{array}{l}.24 \\
-82)\end{array}$ & M 62, & 39 & LUAD & LUAD & | 19, || 18, ||| & 6, IV 8 \\
\hline Chae YK [38] & 2019 & $\begin{array}{l}2015- \\
2016\end{array}$ & USA & 90 & 46 & $\begin{array}{l}\text { Mean } \\
65.7\end{array}$ & $\begin{array}{l}\text { Mean } \\
71.5\end{array}$ & $\begin{array}{l}M \\
36, F \\
54\end{array}$ & $\begin{array}{l}M \\
17, F \\
29\end{array}$ & $\begin{array}{l}\text { LUAD 70, } \\
\text { LUSC 13, } \\
\text { PNE } 5\end{array}$ & $\begin{array}{l}\text { LUAD 29, } \\
\text { LUSC 14, } \\
\text { PNE } 1\end{array}$ & $\begin{array}{l}\text { I-III 16, IV } \\
74\end{array}$ & $\begin{array}{l}\text { I-III 9, IV } \\
37\end{array}$ \\
\hline Chae YK [40] & 2019 & $\begin{array}{l}2013- \\
2016\end{array}$ & USA & 54 & 18 & $\begin{array}{l}\text { Median } 64 \\
\text { range (37- }\end{array}$ & $\begin{array}{l}4.5 \\
-88)\end{array}$ & M 33, & 49 & LUAD 64. L & JSC 11 & $\begin{array}{l}\mathrm{I}-\mathrm{III} 11, \mathrm{IV} \\
43\end{array}$ & $\begin{array}{l}\mathrm{I}-\mathrm{III} 4, \mathrm{IV} \\
14\end{array}$ \\
\hline Singal G [52] & 2019 & $\begin{array}{l}2011- \\
2018\end{array}$ & USA & 1116 & 161 & $\begin{array}{l}\text { Median } 66 \\
(58.0-73 .\end{array}$ & 0), IQR & $\begin{array}{l}\text { M } 195 \\
2109\end{array}$ & & $\begin{array}{l}\text { non-LUSC } \\
726\end{array}$ & 153, LUSC & $\begin{array}{l}\text { | 388, || 309, } \\
2304\end{array}$ & II 846, IV \\
\hline
\end{tabular}

Abbreviations: TMB, tumor mutational burden; $M$, male; $F$, female; LUAD, lung adenocarcinoma; LUSC, lung squamous cell carcinoma; NA, not acquired; ICI, in sequencing; WES, whole exome sequencing; mut, mutation; NSCLC, non-small cell lung cancer; SCLC, small cell lung cancer; PSC, pulmnary sarcomatoid carc disease-free survival; IQR, interquartile range. 


\begin{tabular}{|c|c|c|c|c|c|c|c|c|c|c|c|c|c|}
\hline \multirow{3}{*}{$\begin{array}{l}\text { Fang W [53] } \\
\text { Wang Z [39] }\end{array}$} & \multirow{3}{*}{$\begin{array}{l}2019 \\
2019\end{array}$} & \multirow{3}{*}{$\begin{array}{l}2015- \\
2019 \\
\\
2016- \\
2018\end{array}$} & \multirow{3}{*}{$\begin{array}{l}\text { China } \\
\text { China }\end{array}$} & \multicolumn{2}{|c|}{ Sample size } & \multirow{2}{*}{\multicolumn{2}{|c|}{$\begin{array}{l}\text { Age } \\
\begin{array}{l}\text { Median 54, range } \\
(28-73)\end{array}\end{array}$}} & \multirow{2}{*}{\multicolumn{2}{|c|}{$\begin{array}{l}\text { Gender } \\
\text { M 53, F } 25\end{array}$}} & \multirow{2}{*}{\multicolumn{2}{|c|}{$\begin{array}{l}\text { Tumor histology } \\
\text { LUAD 47, LUSC 24, } \\
\text { others } 7\end{array}$}} & \multirow{2}{*}{\multicolumn{2}{|c|}{$\begin{array}{l}\text { Stage } \\
\text { III 1, IV } 77\end{array}$}} \\
\hline & & & & 49 & 26 & & & & & & & & \\
\hline & & & & 22 & 28 & $\begin{array}{l}\text { Median } \\
56\end{array}$ & $\begin{array}{l}\text { Median } \\
59\end{array}$ & $\begin{array}{l}M \\
12, F \\
10\end{array}$ & $\begin{array}{l}M \\
23, F \\
5\end{array}$ & $\begin{array}{l}\text { LUSC 7, } \\
\text { nonLUSC } \\
15\end{array}$ & $\begin{array}{l}\text { LUSC 12, } \\
\text { nonLUSC } \\
16\end{array}$ & Advanced & Advanced \\
\hline Chun YJ [54] & 2019 & $\begin{array}{l}2014- \\
2016\end{array}$ & Korea & 88 & 104 & \multicolumn{2}{|c|}{$\begin{array}{l}\text { Median } 63 \text {, range } \\
(34-83)\end{array}$} & \multicolumn{2}{|c|}{ M 87, F 114} & LUAD & LUAD & \multicolumn{2}{|c|}{ | 113, || 44, ||| 42, IV 2} \\
\hline $\begin{array}{l}\text { Hellmann } \\
\text { MD [31] }\end{array}$ & 2018 & $\begin{array}{l}2013- \\
2018\end{array}$ & USA & 86 & 47 & \multicolumn{2}{|c|}{$\begin{array}{l}\text { Median 63, range } \\
(29-83)\end{array}$} & NA & NA & SCLC & SCLC & NA & NA \\
\hline $\begin{array}{l}\text { Hellmann } \\
\text { MD [31] }\end{array}$ & 2018 & $\begin{array}{l}2013- \\
2018\end{array}$ & USA & 52 & 26 & \multicolumn{2}{|c|}{$\begin{array}{l}\text { Median 65, range } \\
(37-80)\end{array}$} & NA & NA & SCLC & SCLC & NA & NA \\
\hline $\begin{array}{l}\text { Hellmann } \\
\text { MD [31] }\end{array}$ & 2018 & $\begin{array}{l}2013- \\
2018\end{array}$ & USA & 66 & 34 & \multicolumn{2}{|l|}{ NA } & NA & NA & SCLC & SCLC & NA & NA \\
\hline $\begin{array}{l}\text { Devarakonda } \\
\text { S [55] }\end{array}$ & 2018 & NA & USA & 616 & 292 & \multicolumn{2}{|c|}{$\begin{array}{l}\text { Median } 60 \text {, range } \\
(27-81)\end{array}$} & \multicolumn{2}{|c|}{ M 662, F 246} & \multicolumn{2}{|c|}{ LUAD 375, LUSC 414} & \multicolumn{2}{|c|}{ | 114, || 676, III/IV 114} \\
\hline $\begin{array}{l}\text { Sorotsky H } \\
\text { [27] }\end{array}$ & 2018 & NA & Canada & 74 & & \multicolumn{2}{|c|}{$\leq 6544, \varangle 6430$} & \multicolumn{2}{|c|}{ M 22, F 52} & \multicolumn{2}{|l|}{ NSCLC } & \multicolumn{2}{|c|}{ | 38, || 14, ||I/IV 22} \\
\hline Zheng S [56] & 2018 & $\begin{array}{l}1991- \\
2014\end{array}$ & USA & 75 & 44 & NA & NA & NA & NA & NSCLC & NSCLC & I & I \\
\hline $\begin{array}{l}\text { Owada-Ozaki } \\
\text { Y [57] }\end{array}$ & 2018 & $\begin{array}{l}2013- \\
2016\end{array}$ & Japan & 44 & 46 & \multicolumn{2}{|c|}{$\begin{array}{l}\text { Median 70, range } \\
(40-87)\end{array}$} & \multicolumn{2}{|c|}{ M 63, F 27} & \multicolumn{2}{|c|}{ LUAD 63, LUSC 27} & \multicolumn{2}{|c|}{ | 67, || 12, ||| 11} \\
\hline Rizvi H [58] & 2018 & $\begin{array}{l}2011- \\
2017\end{array}$ & USA & 119 & 121 & \multicolumn{2}{|c|}{$\begin{array}{l}\text { Median } 66 \text {, range } \\
(22-92)\end{array}$} & \multicolumn{2}{|c|}{ M 118, F 122} & \multicolumn{2}{|c|}{$\begin{array}{l}\text { LUAD 186, LUSC } 34 \text {, } \\
\text { Others } 20\end{array}$} & Advanced & Advanced \\
\hline Rizvi H [58] & 2018 & $\begin{array}{l}2011- \\
2017\end{array}$ & USA & 315 & 272 & NA & NA & NA & NA & LUAD & LUAD & Advanced & Advanced \\
\hline $\begin{array}{l}\text { Hellmann } \\
\text { MD [59] }\end{array}$ & 2018 & $\begin{array}{l}2014- \\
2015\end{array}$ & USA & 38 & 37 & $\begin{array}{l}\text { Median } \\
66, \\
\text { range } \\
\left(43^{-}\right. \\
85)\end{array}$ & $\begin{array}{l}\text { Median } \\
65, \\
\text { range } \\
\left(42^{-}\right. \\
87)\end{array}$ & $\begin{array}{l}M \\
17, F \\
21\end{array}$ & $\begin{array}{l}M \\
20, F \\
17\end{array}$ & $\begin{array}{l}\text { LUSC 7, } \\
\text { nonLUSC } \\
31\end{array}$ & $\begin{array}{l}\text { LUSC9, } \\
\text { nonLUSC } \\
28\end{array}$ & $\begin{array}{l}\text { IIIB } 6, \text { IV } \\
32\end{array}$ & $\begin{array}{l}\operatorname{IIIB} 3, \text { IV } \\
34\end{array}$ \\
\hline Miao D [60] & 2018 & NA & USA & 28 & 28 & $\begin{array}{l}\text { Median } \\
61, \\
\text { range } \\
(41- \\
78)\end{array}$ & $\begin{array}{l}\text { Median } \\
62 \text {, } \\
\text { range } \\
(54- \\
72)\end{array}$ & $\begin{array}{l}M \\
11, F \\
17\end{array}$ & $\begin{array}{l}M \\
13, F \\
15\end{array}$ & $\begin{array}{l}\text { LUAD 26, } \\
\text { LUSC } 2\end{array}$ & $\begin{array}{l}\text { LUAD 21, } \\
\text { LUSC 5, } \\
\text { Others } 2\end{array}$ & NA & NA \\
\hline $\begin{array}{l}\text { Kowanetz M } \\
\text { [61] }\end{array}$ & 2017 & 2015 & USA & 51 & 51 & NA & NA & NA & NA & NSCLC & NSCLC & Advanced & Advanced \\
\hline $\begin{array}{l}\text { Kowanetz M } \\
\text { [61] }\end{array}$ & 2017 & 2015 & USA & 51 & 186 & NA & NA & NA & NA & NSCLC & NSCLC & Advanced & Advanced \\
\hline $\begin{array}{l}\text { Wungki P } \\
\text { [62] }\end{array}$ & 2017 & NA & USA & 36 & & NA & & NA & & NSCLC & NSCLC & Advanced & Advanced \\
\hline Xiao D [63] & 2016 & NA & China & 288 & 47 & \65 228, & 65107 & M 183, & F 152 & LUAD & LUAD & | 82, || 69, || & 54, IV 29 \\
\hline Rizvi NA [64] & 2015 & NA & USA & 17 & 17 & $\begin{array}{l}\text { Median } \\
60, \\
\text { range } \\
(41- \\
80)\end{array}$ & $\begin{array}{l}\text { Median } \\
63, \\
\text { range } \\
(56- \\
78)\end{array}$ & $\begin{array}{l}M \\
10, F \\
7\end{array}$ & $\begin{array}{l}\text { M 6, } \\
\text { F } 11\end{array}$ & $\begin{array}{l}\text { LUAD 14, } \\
\text { LUSC } 3\end{array}$ & $\begin{array}{l}\text { LUAD 15, } \\
\text { LUSC 1, } \\
\text { Others } 1\end{array}$ & IV & IV \\
\hline
\end{tabular}

Abbreviations: TMB, tumor mutational burden; M, male; F, female; LUAD, lung adenocarcinoma; LUSC, lung squamous cell carcinoma; NA, not acquired; ICI, in sequencing; WES, whole exome sequencing; mut, mutation; NSCLC, non-small cell lung cancer; SCLC, small cell lung cancer; PSC, pulmnary sarcomatoid carc disease-free survival; IQR, interquartile range.

\subsubsection{Meta-analysis Results}

A total of 22 articles with 28 studies evaluated OS, and 22 articles with 25 studies evaluated PFS. The HRs and $95 \% \mathrm{Cl}$ were pooled using the random-effects models throughout the analysis. On the whole, high-TMB group lung cancer had better PFS compared to low-TMB group (Fig. 8B, $\mathrm{P}=0.001)$. However, no 
significant difference in OS was found between the high TMB group and the low TMB group (Fig. 8A, P=0.830).

The above total lung cancer patients include different types of lung cancer, different treatment methods, different TMB detection methods, and different test samples. To explore the impact of these factors on results, we conducted a series of subgroup analyses. In a subgroup analysis based on whether to receive ICls, we found that among patients receiving ICls, the high TMB group had better OS (Fig. 9A, P<0.001) and PFS (Fig. 10A, P<0.001). However, in the non-ICI subgroup, the OS in the high TMB group was worse compared with the low TMB group (Fig. 9A, $P=0.014$ ), and there was no significant difference in PFS between the two groups (Fig. 9A, $\mathrm{P}=0.464)$. In the SCLC subgroup, the OS (Fig. 9B, $\mathrm{P}=0.052$ ) and PFS (Fig. 10B, $\mathrm{P}<0.001)$ in the high TMB group are better than those in the low TMB group, but in NSCLC subgroup, there is no significant difference between the high and low TMB groups (Fig. 9B and Fig. 10B). NSCLC was further divided into LUAD and LUSC. The results showed that in the LUAD subgroup, the OS (Fig. 9C, P<0.001) and PFS (Fig. 10C, P = 0.051) of the high TMB group were worse than the low TMB group. However, in the LUSC subgroup, there was no significant difference between the high and low TMB groups (Fig. $9 \mathrm{C}$ and Fig. 10C). Considering that immunotherapy has a greater impact on the clinical prognosis of TMB, we combined the tumor pathological type and ICls for subgroup analysis. Results showed that in the NSCLC subgroup receiving ICls, high TMB was associated with better OS (Fig. 9D, $\mathrm{P}=0.006$ ) and PFS (Fig. 10D, P<0.001), with similar results in the SCLC subgroup (OS, Fig. 9D, P = 0.065; PFS, Fig. 10D, P<0.001). Prognostic effect of TMB diminished in NSCLC and SCLC subgroups not receiving ICls (Fig. 9D and Fig. 10D). High-TMB was a poor prognostic indicator in LUAD subgroup not receiving ICI (OS, Fig. 9D, P<0.001; PFS, Fig. 10D, P = 0.035). However, in a LUAD subgroup not receiving ICl, TMB had no significant prognostic value (Fig. 9D and Fig. 10D).

In the subgroup analysis based on the detection method, we found that targeted NGS and WES did not significantly affect the indicator role of TMB in OS (Fig. 11A). However, different detection methods had a greater impact on the prognosis of TMB in PFS. In the targeted NGS subgroup, high TMB predicted higher PFS (Fig. 12A, P < 0.001). In the WES subgroup, TMB does not show an indicator effect (Fig. 12A). In the subgroup analysis of detection methods combined with immunotherapy, whether using the targeted NGS or WES methods, in the subgroup receiving ICl, high TMB tended to predict a better prognosis. Still in the subgroup not receiving ICl, high TMB tended to have a poor prognosis (Fig. 11B and Fig. 12B). Then we performed a subgroup analysis based on different test samples. In the subgroup tested using blood samples, the high TMB group had worse OS than the low TMB group (Fig. 11C, $\mathrm{P}=0.027$ ). In the subgroup detected using tumor tissue samples, the high TMB group had better PFS than the low TMB group (Fig. 12C, P = 0.001). Subgroup analysis was further performed by combining test samples and immunotherapy. The results showed that high-TMB had a better prognosis in the subgroup that used tissue detection and received ICls (OS, Fig. 11D, P<0.001; PFS, Fig. 12D, P<0.001). However, in the subgroup that provided tissue testing but did not receive ICl, high TMB tended to have a worse prognosis (OS, Fig. 11D, $P=0.045)$.

\subsubsection{Effect of TMB on EGFR-mutation or EGFR-TKIs treated lung cancer}

Four articles reported the role of TMB in EGFR-mutant or EGFR-TKIs treated lung cancer [11, 25-27]. Machael Offin et al. studied EGFR exon19del or L858R mutant LUAD treated with EGFR-TKIs [11]. By using EGFR wild-type LUAD as a control group, they found that the TMB value of EGFR-mutated lung adenocarcinoma was lower than that of EGFR wild-type lung adenocarcinoma patients, and TMB was a poor prognostic factor in metastatic EGFR mutant LUAD treated with EGFR-TKIs [11]. Yanhui Chen et al. enrolled into two cohorts of LUAD and LUSC, and found that the TMB value in LUAD was lower than that in LUSC [26]. There were only two patients with EGFR mutations in the LUSC group, accounting for $4 \%$. In the LUAD group, there were 58 patients with EGFR mutations, accounting for $43 \%$. In EGFR wild-type LUAD, TMB did not correlate with survival outcomes $(P=0.484)$. Still, in mixed EGFR mutant and wild-type patients, TMB was closer to a worse prognostic factor $(P=0.062)$, which means that TMB tended to negatively related to survival in patients with EGFR mutant LUAD [26]. Through the study of EGFR-mutant advanced LUAD, Xiaodong Jiao et al. found that TMB was negatively correlated with OS in EGFRmutant LUAD patients [25]. H. Sorotsky et al. studied early NSCLC with surgical resection of EGFR mutations and found that TMB is associated with shorter survival but has little to do with disease relapse [27].

\subsubsection{Publication Bias And Sensitivity Analysis}

According to Begg's and Egger's test, the publication bias of OS (Begg's $P=0.767$; Egger's $P=0.765)$ and PFS (Begg's $P=0.528$; Egger's $P=0.591)$ was not significant. By omitting each study one by one, no single study with a significant impact on the combined results was found, which means that the metaanalysis results were reliable.

\section{Discussion}

With the development of sequencing technology, TMB has become an essential clinical indicator [28, 29]. Currently, TMB is mainly studied as a predictive biomarker for ICls treatment response [15,30]. Recent studies have concluded that in patients with higher TMB, the survival benefit of patients receiving ICls is better than that of patients receiving chemotherapy alone, but in patients with low TMB, the survival benefit of ICls is not statistically significant [17, 18]. In this study, we did not group patients according to TMB value, but grouped according to lung cancer types, treatment methods, detection methods, test samples. We found that high TMB in the immunotherapy group was an excellent prognostic indicator, which corresponded to high TMB as a marker of immunotherapy responsiveness. But in NSCLC patients who receive chemo-radiotherapy or targeted therapy but not ICls treatment, high TMB is associated with a worse prognosis, which suggests that TMB itself may be a poor prognostic indicator in NSCLC.

According to the type of lung cancer, TMB is a good prognostic indicator in SCLC, but a poor prognosis indicator in NSCLC. Immunotherapy enhances the role of TMB in SCLC to indicate better prognosis, but reverses the role of TMB in NSCLC, making TMB from a poor prognostic indicator to a better prognostic indicator. The beneficial effect of high TMB on long-term survival of NSCLC and SCLC patients treated with ICls validates the therapeutic value of immunotherapy for patients with high TMB [31, 32]. In LUAD, including EGFR mutated and EGFR-TKIs treated LUAD, high TMB is a poor prognostic indicator. Fortunately, it was found in this study that TMB values are usually lower in LUAD patients, especially those with EGFR mutations. However, for LUAD patients 
with EGFR mutations and high TMB, whether targeted therapy is adequate and whether additional or alternative immunotherapy is needed should be further studied [33].

In terms of different detection methods, the detection results of WES and targeted NGS are consistent, and have no significant impact on the clinical effect evaluation of TMB. However, the TMB value detected by WES is higher than that detected by targeted NGS, which may be because the WES method contains more mutation sites. In clinical applications, targeted NGS is obviously more suitable for clinical work than WES because it saves testing resources and time [34]. At present, different types and quantities of gene panels are used for targeted NGS in different institutions, which results in various TMB benchmark values detected by various institutions. Exploring gene panels suitable for specific tumors in specific populations will help promote the better clinical application of TMB [35].

Compared with circulating tumor DNA (ctDNA) testing, tissue testing is still the current mainstream method [36]. Blood testing has the advantage of convenient material collection, but the testing technology needs further investigation [37]. In this study, a total of three articles with four studies [26, 38, 39] used blood samples, and the results were heterogeneous so that no credible conclusions could be drawn about the validity of the blood test results. In this meta-analysis, we observed that Chae YK et al. used tumor tissue and blood tissue to detect TMB in NSCLC, respectively, and obtained almost opposite conclusions $[38,40]$. Chae YK et al. used tumor tissue to detect TMB in patients with NSCLC receiving ICls and observed that high TMB predicting longer OS $(\mathrm{HR}=0.10,95 \% \mathrm{Cl} 0.01-0.76, \mathrm{P}=0.026)$ [40]. On the other hand, Chae YK et al. used blood samples to test TMB and found that high TMB was associated with poor OS ( $\mathrm{HR}=6.0,95 \% \mathrm{Cl} 1,3-27.1, \mathrm{P}<0.01)$ and PFS $(\mathrm{HR}=5.6,95 \% \mathrm{Cl} 1.3-24.6, \mathrm{P}<0.01)$ among NSCLC patients receiving ICls [38]. However, in the subsequent verification group, Chae $\mathrm{YK}$ et al. found that the ctDNA TMB had no significant effect on OS $(\mathrm{HR}=2.8,95 \% \mathrm{Cl} 0.56-14.4, \mathrm{P}=0.17)$ and $\mathrm{PFS}(\mathrm{HR}=$ $1.1,95 \% \mathrm{Cl} 0.31-4.2, \mathrm{P}>0.05)$ in NSCLC patients receiving ICls [38]. The clinical evaluation of TMB using blood specimens from the same institution differs greatly from the results obtained using tumor tissues, and the results of blood specimens show some instability. The conclusion drawn from this blood specimen is also inconsistent with the mainstream view that the high TMB patients should have better survival benefits in NSCLC patients treated with ICls $[17,18]$. The study author Chae YK et al. also previously found a low correlation in ctDNA TMB and tissue TMB in paired patient samples [41]. In addition to the limitations of ctDNA detection technology, ctDNA TMB may have different clinical significance than tissue TMB. The relationship between ctDNA TMB and tissue TMB and its clinical significance remains to be further explored in larger sample studies.

Compared with the current mainstream researches [42], we focused on the prognostic role of TMB in different lung patient groups, supplementing the role of TMB in traditional chemoradiotherapy and targeted therapy populations. Despite our comprehensive analysis, there are some limitations be recognized. First, the studies included in our report were mainly observational cohort studies, which might provide weaker statistical power. Fortunately, the measurement of TMB values was objective, and most studies were grouped by the median, so the endpoint events were less affected by selective bias. Some of the included studies were secondary analyses of clinical trial results, which could also increase the validity of the results. Second, several HRs and its $95 \% \mathrm{Cl}$ were calculated by extracting data from the Kaplan-Meier curves, which may inevitably lead to statistical bias. Third, the baseline of TMB detected by different testing platforms was different, which might bring bias to combined results. Each study distinguished high and low TMB groups according to the relative value of TMB, which could avoid bias caused by different TMB baselines. The accepted standardization methods of cross-platform TMB results are still one of the critical directions for clinical application of TMB $[43,44]$.

\section{Conclusion}

In this study, we found that TMB is an excellent prognostic indicator in lung cancer patients receiving immunotherapy. Still, it is a poor prognostic indicator in patients with lung cancer receiving traditional chemo-radiotherapy or targeted therapy. EGFR mutation status in patients with lung adenocarcinoma is associated with lower TMB, but some patients have both EGFR mutation and high TMB status. How to choose immunotherapy or targeted therapy for lung adenocarcinoma patients with both EGFR mutation and high TMB needs to be further studied in well-designed randomized controlled clinical trials.

\section{Abbreviations}

TMB, tumor mutational burden; NSCLC, non-small cell lung cancer; SCLC, small cell lung cancer; LUAD, lung adenocarcinoma; LUSC, lung Squamous cell carcinoma; ICl, immune checkpoint inhibitor; NGS, next-generation sequencing; WES, whole exome sequencing; MSI, microsatellite instability; MMR, mismatch repair deficiency; TKI, tyrosine kinase inhibitor; OS, overall survival; PFS, progression-free survival; DFS, disease-free survival; IQR, interquartile range; ES, effect size; HR, hazard ratio; $\mathrm{Cl}$, confidence interval; ctDNA, circulating tumor DNA.

\section{Declarations}

\section{Ethics approval and consent to participate}

Not applicable. This is a secondary analysis of gene sequencing data and literature.

\section{Consent for publication}

All authors agree to publish.

\section{Availability of data and materials}

Single nucleotide mutation, gene expression and patient follow-up data were obtained from The Cancer Genome Atlas (TCGA) database (http://portal.gdc.cancer.gov/). 


\section{Competing interests}

The authors declare no conflict of interest.

Funding

This study was supported by the Zhejiang Traditional Chinese Medicine Scientific Research Fund Program (2017ZA084), Zhejiang Medical and Health Science and Technology Project (2019328069), Zhejiang Natural Science Foundation (LY18H160021), Zhejiang Province Lung Cancer Diagnosis and Treatment Technology Research Center, and Zhejiang Province Traditional Chinese Medicine (Integrated Traditional Chinese and Western Medicine) Key Disciplines (2017 -XK-A33). The funder(s) did not participate in this study.

\section{Authors' Contributions}

YW and $\mathrm{JH}$ are involved in project design, improvement and finalization. LZ participates in the project design and undertakes the specific work of the research. $\mathrm{PX}, \mathrm{ZW}$, and LY participate in literature search, literature data extraction, and discussion of disputed data. WL participates in project improvement, dispute data discussion, article revision and finalization. All authors have read and approved the manuscript.

\section{Acknowledgments}

Not applicable.

\section{Authors' Information}

YW and $\mathrm{JH}$ are cooperating medical colleagues, working together to build an integrated diagnosis and treatment system for lung cancer patients. They have accumulated rich experience in neoadjuvant immunotherapy and neoadjuvant therapy for lung cancer patients. LZ, PX, ZW, and LY are all students of JH. They are engaged in the diagnosis and treatment of lung cancer patients and carry out basic research on lung cancer. WL is the administrator of JH's laboratory, mainly responsible for the laboratory's daily work and student guidance.

\section{References}

1. Siegel RL, Miller KD, Jemal A: Cancer statistics, 2019. CA: a cancer journal for clinicians 2019, 69(1):7-34.

2. Chen Z, Fillmore CM, Hammerman PS, Kim CF, Wong K-K: Non-small-cell lung cancers: a heterogeneous set of diseases. Nature reviews Cancer 2014, 14(8):535-546.

3. Kalemkerian GP, Schneider BJ: Advances in Small Cell Lung Cancer. Hematology/oncology clinics of North America 2017, 31(1):143-156.

4. Reck M, Rabe KF: Precision Diagnosis and Treatment for Advanced Non-Small-Cell Lung Cancer. The New England journal of medicine 2017, 377(9):849861.

5. Rossi A, Tay R, Chiramel J, Prelaj A, Califano R: Current and future therapeutic approaches for the treatment of small cell lung cancer. Expert review of anticancer therapy 2018, 18(5):473-486.

6. Herbst RS, Morgensztern D, Boshoff C: The biology and management of non-small cell lung cancer. Nature 2018, 553(7689):446-454.

7. Soda M, Choi YL, Enomoto M, Takada S, Yamashita Y, Ishikawa S, Fujiwara S-i, Watanabe H, Kurashina K, Hatanaka H et al: Identification of the transforming EML4-ALK fusion gene in non-small-cell lung cancer. Nature 2007, 448(7153):561-566.

8. Lynch TJ, Bell DW, Sordella R, Gurubhagavatula S, Okimoto RA, Brannigan BW, Harris PL, Haserlat SM, Supko JG, Haluska FG et al: Activating mutations in the epidermal growth factor receptor underlying responsiveness of non-small-cell lung cancer to gefitinib. The New England journal of medicine 2004 , 350(21):2129-2139.

9. Rekhtman N, Paik PK, Arcila ME, Tafe LJ, Oxnard GR, Moreira AL, Travis WD, Zakowski MF, Kris MG, Ladanyi M: Clarifying the spectrum of driver oncogene mutations in biomarker-verified squamous carcinoma of lung: lack of EGFR/KRAS and presence of PIK3CAVAKT1 mutations. Clinical cancer research : an official journal of the American Association for Cancer Research 2012, 18(4):1167-1176.

10. Heigener DF, Reck M: Advanced non-small cell lung cancer: the role of PD-L1 inhibitors. Journal of thoracic disease 2018, 10(Suppl 13):S1468-S1473.

11. Offin M, Rizvi H, Tenet M, Ni A, Sanchez-Vega F, Li BT, Drilon A, Kris MG, Rudin CM, Schultz N et al: Tumor Mutation Burden and Efficacy of EGFR-Tyrosine Kinase Inhibitors in Patients with EGFR-Mutant Lung Cancers. Clinical cancer research : an official journal of the American Association for Cancer Research 2019, 25(3):1063-1069.

12. Rozenblum AB, llouze M, Dudnik E, Dvir A, Soussan-Gutman L, Geva S, Peled N: Clinical Impact of Hybrid Capture-Based Next-Generation Sequencing on Changes in Treatment Decisions in Lung Cancer. Journal of thoracic oncology : official publication of the International Association for the Study of Lung Cancer 2017, 12(2):258-268.

13. Bernicker EH, Miller RA, Cagle PT: Biomarkers for Selection of Therapy for Adenocarcinoma of the Lung. Journal of oncology practice 2017, 13(4):221227.

14. Goodman AM, Kato S, Bazhenova L, Patel SP, Frampton GM, Miller V, Stephens PJ, Daniels GA, Kurzrock R: Tumor Mutational Burden as an Independent Predictor of Response to Immunotherapy in Diverse Cancers. Molecular cancer therapeutics 2017, 16(11):2598-2608.

15. Yarchoan M, Hopkins A, Jaffee EM: Tumor Mutational Burden and Response Rate to PD-1 Inhibition. The New England journal of medicine 2017, 377(25):2500-2501. 
16. Alborelli I, Leonards K, Rothschild SI, Leuenberger LP, Savic Prince S, Mertz KD, Poechtrager S, Buess M, Zippelius A, Läubli H et al: Tumor mutational burden assessed by targeted NGS predicts clinical benefit from immune checkpoint inhibitors in non-small cell lung cancer. The Journal of pathology 2020, 250(1):19-29.

17. Kim JY, Kronbichler A, Eisenhut M, Hong SH, van der Vliet HJ, Kang J, Shin JI, Gamerith G: Tumor Mutational Burden and Efficacy of Immune Checkpoint Inhibitors: A Systematic Review and Meta-Analysis. Cancers 2019, 11(11):1798.

18. Klempner SJ, Fabrizio D, Bane S, Reinhart M, Peoples T, Ali SM, Sokol ES, Frampton G, Schrock AB, Anhorn R et al: Tumor Mutational Burden as a Predictive Biomarker for Response to Immune Checkpoint Inhibitors: A Review of Current Evidence. The oncologist 2020, 25(1):e147-e159.

19. Yu Y, Zeng D, Ou Q, Liu S, Li A, Chen Y, Lin D, Gao Q, Zhou H, Liao W et al: Association of Survival and Immune-Related Biomarkers With Immunotherapy in Patients With Non-Small Cell Lung Cancer: A Meta-analysis and Individual Patient-Level Analysis. JAMA network open 2019, 2(7):e196879-e196879.

20. Mayakonda A, Lin D-C, Assenov Y, Plass C, Koeffler HP: Maftools: efficient and comprehensive analysis of somatic variants in cancer. Genome Research 2018, 28(11):1747-1756.

21. Moher D, Liberati A, Tetzlaff J, Altman DG, Group P: Preferred reporting items for systematic reviews and meta-analyses: the PRISMA statement. BMJ (Clinical research ed) 2009, 339:b2535-b2535.

22. Tierney JF, Stewart LA, Ghersi D, Burdett S, Sydes MR: Practical methods for incorporating summary time-to-event data into meta-analysis. Trials 2007, 8:16-16.

23. Stang A: Critical evaluation of the Newcastle-Ottawa scale for the assessment of the quality of nonrandomized studies in meta-analyses. European journal of epidemiology 2010, 25(9):603-605.

24. Higgins JPT, Thompson SG, Deeks JJ, Altman DG: Measuring inconsistency in meta-analyses. BMJ (Clinical research ed) 2003, 327(7414):557-560.

25. Jiao X-D, He X, Qin B-D, Liu K, Wu Y, Liu J, Hou T, Zang Y-S: The prognostic value of tumor mutation burden in EGFR-mutant advanced lung adenocarcinoma, an analysis based on cBioPortal data base. Journal of thoracic disease 2019, 11(11):4507-4515.

26. Chen Y, Liu Q, Chen Z, Wang Y, Yang W, Hu Y, Han W, Zeng H, Ma H, Dai J et al: PD-L1 expression and tumor mutational burden status for prediction of response to chemotherapy and targeted therapy in non-small cell lung cancer. Journal of experimental \& clinical cancer research : CR 2019, 38(1):193193.

27. Sorotsky H, Cabanero M, Moskovitz M, Weiss J, Pintilie M, Leighl N, Bradbury P, Liu G, Kia A, Pugh T et al: P3. 03-29 The Prognostic Effect of Tumor Mutation Burden and Smoking History in Resected EGFR Mutant Non-Small Cell Lung Cancer. Journal of Thoracic Oncology 2018, $13(10)$ :S921.

28. Chalmers ZR, Connelly CF, Fabrizio D, Gay L, Ali SM, Ennis R, Schrock A, Campbell B, Shlien A, Chmielecki J et al: Analysis of 100,000 human cancer genomes reveals the landscape of tumor mutational burden. Genome Medicine 2017, 9(1):34-34.

29. Bhangoo MS, Boasberg P, Mehta P, Elvin JA, Ali SM, Wu W, Klempner SJ: Tumor Mutational Burden Guides Therapy in a Treatment Refractory POLEMutant Uterine Carcinosarcoma. The oncologist 2018, 23(5):518-523.

30. Samstein RM, Lee C-H, Shoushtari AN, Hellmann MD, Shen R, Janjigian YY, Barron DA, Zehir A, Jordan EJ, Omuro A et al: Tumor mutational load predicts survival after immunotherapy across multiple cancer types. Nature genetics 2019, 51(2):202-206.

31. Hellmann MD, Callahan MK, Awad MM, Calvo E, Ascierto PA, Atmaca A, Rizvi NA, Hirsch FR, Selvaggi G, Szustakowski JD et al: Tumor Mutational Burden and Efficacy of Nivolumab Monotherapy and in Combination with Ipilimumab in Small-Cell Lung Cancer. Cancer Cel/ 2018, 33(5):853-861.e854.

32. Hellmann MD, Ciuleanu T-E, Pluzanski A, Lee JS, Otterson GA, Audigier-Valette C, Minenza E, Linardou H, Burgers S, Salman P et al: Nivolumab plus Ipilimumab in Lung Cancer with a High Tumor Mutational Burden. The New England journal of medicine 2018, 378(22):2093-2104.

33. Saito M, Suzuki H, Kono K, Takenoshita S, Kohno T: Treatment of lung adenocarcinoma by molecular-targeted therapy and immunotherapy. Surgery today 2018, 48(1):1-8.

34. Fancello L, Gandini S, Pelicci PG, Mazzarella L: Tumor mutational burden quantification from targeted gene panels: major advancements and challenges. Journal for Immunotherapy of Cancer 2019, 7(1):183-183.

35. Endris V, Buchhalter I, Allgäuer M, Rempel E, Lier A, Volckmar A-L, Kirchner M, von Winterfeld M, Leichsenring J, Neumann 0 et al: Measurement of tumor mutational burden (TMB) in routine molecular diagnostics: in silico and real-life analysis of three larger gene panels. International journal of cancer 2019 , 144(9):2303-2312.

36. Yang N, Li Y, Liu Z, Qin H, Du D, Cao X, Cao X, Li J, Li D, Jiang B et al: The characteristics of ctDNA reveal the high complexity in matching the corresponding tumor tissues. BMC cancer 2018, 18(1):319-319.

37. Fenizia F, Pasquale R, Roma C, Bergantino F, lannaccone A, Normanno N: Measuring tumor mutation burden in non-small cell lung cancer: tissue versus liquid biopsy. Translational Lung Cancer Research 2018, 7(6):668-677.

38. Chae YK, Davis AA, Agte S, Pan A, Simon NI, lams WT, Cruz MR, Tamragouri K, Rhee K, Mohindra N et al: Clinical Implications of Circulating Tumor DNA Tumor Mutational Burden (ctDNA TMB) in Non-Small Cell Lung Cancer. The oncologist 2019, 24(6):820-828.

39. Wang Z, Duan J, Cai S, Han M, Dong H, Zhao J, Zhu B, Wang S, Zhuo M, Sun J et al: Assessment of Blood Tumor Mutational Burden as a Potential Biomarker for Immunotherapy in Patients With Non-Small Cell Lung Cancer With Use of a Next-Generation Sequencing Cancer Gene Panel. JAMA Oncology 2019, 5(5):696-702.

40. Chae YK, Davis AA, Raparia K, Agte S, Pan A, Mohindra N, Villaflor V, Giles F: Association of Tumor Mutational Burden With DNA Repair Mutations and Response to Anti-PD-1/PD-L1 Therapy in Non-Small-Cell Lung Cancer. Clinical lung cancer 2019, 20(2):88-96.e86.

41. Davis AA, Chae YK, Agte S, Pan A, Simon NI, Taxter TJ, Behdad A, Carneiro BA, Cristofanilli M, Giles FJ: Comparison of tumor mutational burden (TMB) across tumor tissue and circulating tumor DNA (ctDNA). Journal of Clinical Oncology 2017, 35(15_suppl):e23028-e23028. 
42. Berland L, Heeke S, Humbert O, Macocco A, Long-Mira E, Lassalle S, Lespinet-Fabre V, Lalvée S, Bordone O, Cohen C et al: Current views on tumor mutational burden in patients with non-small cell lung cancer treated by immune checkpoint inhibitors. Journal of thoracic disease 2019, 11(Suppl 1):S71-S80.

43. Stenzinger A, Allen JD, Maas J, Stewart MD, Merino DM, Wempe MM, Dietel M: Tumor mutational burden standardization initiatives: Recommendations for consistent tumor mutational burden assessment in clinical samples to guide immunotherapy treatment decisions. Genes, chromosomes \& cancer 2019, 58(8):578-588.

44. Vokes NI, Liu D, Ricciuti B, Jimenez-Aguilar E, Rizvi H, Dietlein F, He MX, Margolis CA, Elmarakeby HA, Girshman J et al: Harmonization of Tumor Mutational Burden Quantification and Association With Response to Immune Checkpoint Blockade in Non-Small-Cell Lung Cancer. JCO precision oncology 2019, 3:10.1200/P0.1219.00171.

45. Ricciuti B, Kravets S, Dahlberg SE, Umeton R, Albayrak A, Subegdjo SJ, Johnson BE, Nishino M, Sholl LM, Awad MM: Use of targeted next generation sequencing to characterize tumor mutational burden and efficacy of immune checkpoint inhibition in small cell lung cancer. Journal for Immunotherapy of Cancer 2019, 7(1):87-87

46. Zhou M, Fan J, Li Z, Li P, Sun Y, Yang Y, Zhou X, Wang J, Wang Y, Qi H et al: Prognostic impact of tumor mutation burden and the mutation in KIAA1211 in small cell lung cancer. Respiratory Research 2019, 20(1):248-248.

47. Heeke S, Benzaquen J, Long-Mira E, Audelan B, Lespinet V, Bordone O, Lalvée S, Zahaf K, Poudenx M, Humbert O et al: In-house Implementation of Tumor Mutational Burden Testing to Predict Durable Clinical Benefit in Non-small Cell Lung Cancer and Melanoma Patients. Cancers 2019, 11(9):1271.

48. Jiang T, Shi J, Dong Z, Hou L, Zhao C, Li X, Mao B, Zhu W, Guo X, Zhang H et al: Genomic landscape and its correlations with tumor mutational burden, PD-L1 expression, and immune cells infiltration in Chinese lung squamous cell carcinoma. Journal of Hematology \& Oncology 2019, 12(1):75-75.

49. Ready N, Hellmann MD, Awad MM, Otterson GA, Gutierrez M, Gainor JF, Borghaei H, Jolivet J, Horn L, Mates M et al: First-Line Nivolumab Plus Ipilimumab in Advanced Non-Small-Cell Lung Cancer (CheckMate 568): Outcomes by Programmed Death Ligand 1 and Tumor Mutational Burden as Biomarkers. Journal of clinical oncology : official journal of the American Society of Clinical Oncology 2019, 37(12):992-1000.

50. Yu H, Chen Z, Ballman KV, Watson MA, Govindan R, Lanc I, Beer DG, Bueno R, Chirieac LR, Chui MH et al: Correlation of PD-L1 Expression with Tumor Mutation Burden and Gene Signatures for Prognosis in Early-Stage Squamous Cell Lung Carcinoma. Journal of thoracic oncology : official publication of the International Association for the Study of Lung Cancer 2019, 14(1):25-36.

51. Wang C, Liang H, Lin C, Li F, Xie G, Qiao S, Shi X, Deng J, Zhao X, Wu K et al: Molecular Subtyping and Prognostic Assessment Based on Tumor Mutation Burden in Patients with Lung Adenocarcinomas. International journal of molecular sciences 2019, 20(17):4251.

52. Singal G, Miller PG, Agarwala V, Li G, Kaushik G, Backenroth D, Gossai A, Frampton GM, Torres AZ, Lehnert EM et al: Association of Patient Characteristics and Tumor Genomics With Clinical Outcomes Among Patients With Non-Small Cell Lung Cancer Using a Clinicogenomic Database. Jama 2019, 321(14):1391-1399.

53. Fang W, Ma Y, Yin JC, Hong S, Zhou H, Wang A, Wang F, Bao H, Wu X, Yang Y et al: Comprehensive Genomic Profiling Identifies Novel Genetic Predictors of Response to Anti-PD-(L)1 Therapies in Non-Small Cell Lung Cancer. Clinical cancer research : an official journal of the American Association for Cancer Research 2019, 25(16):5015-5026.

54. Chun YJ, Choi JW, Hong MH, Jung D, Son H, Cho EK, Min YJ, Kim S-W, Park K, Lee SS et al: Molecular characterization of lung adenocarcinoma from Korean patients using next generation sequencing. PloS one 2019, 14(11):e0224379-e0224379.

55. Devarakonda S, Rotolo F, Tsao M-S, Lanc I, Brambilla E, Masood A, Olaussen KA, Fulton R, Sakashita S, McLeer-Florin A et al: Tumor Mutation Burden as a Biomarker in Resected Non-Small-Cell Lung Cancer. Journal of clinical oncology : official journal of the American Society of Clinical Oncology 2018, 36(30):2995-3006.

56. Zheng S, Xia J, Zeng F, Huang L, Li F, Zhu H, Liao G, Lin Z, Zhou H: P021 The Impact of TP53 Mutation and Tumor Mutation Number on Outcomes in Patients with Stage I Non Small Cell Lung Cancer. Journal of Thoracic Oncology 2018, 13(12):S1058.

57. Owada-Ozaki Y, Muto S, Takagi H, Inoue T, Watanabe Y, Fukuhara M, Yamaura T, Okabe N, Matsumura Y, Hasegawa T et al: Prognostic Impact of Tumor Mutation Burden in Patients With Completely Resected Non-Small Cell Lung Cancer: Brief Report. Journal of thoracic oncology : official publication of the International Association for the Study of Lung Cancer 2018, 13(8):1217-1221.

58. Rizvi H, Sanchez-Vega F, La K, Chatila W, Jonsson P, Halpenny D, Plodkowski A, Long N, Sauter JL, Rekhtman N et al: Molecular Determinants of Response to Anti-Programmed Cell Death (PD)-1 and Anti-Programmed Death-Ligand 1 (PD-L1) Blockade in Patients With Non-Small-Cell Lung Cancer Profiled With Targeted Next-Generation Sequencing. Journal of clinical oncology : official journal of the American Society of Clinical Oncology 2018, 36(7):633-641.

59. Hellmann MD, Nathanson T, Rizvi H, Creelan BC, Sanchez-Vega F, Ahuja A, Ni A, Novik JB, Mangarin LMB, Abu-Akeel M et al: Genomic Features of Response to Combination Immunotherapy in Patients with Advanced Non-Small-Cell Lung Cancer. Cancer Cell 2018, 33(5):843-852.e844.

60. Miao D, Margolis CA, Vokes NI, Liu D, Taylor-Weiner A, Wankowicz SM, Adeegbe D, Keliher D, Schilling B, Tracy A et al: Genomic correlates of response to immune checkpoint blockade in microsatellite-stable solid tumors. Nature genetics 2018, 50(9):1271-1281.

61. Marcin K, Wei Z, Shames D: Tumor mutation burden (TMB) is associated with improved efficacy of atezolizumab In 1L and 2L+ NSCLC patients. Journal of thoracic oncology : official publication of the International Association for the Study of Lung Cancer 2016, 4(Suppl):abstr OA20. 01.

62. Park W, Lopes G, Kwon D, Florou V, Chae Y, Warsch J, Ishkanian A, Jahanzeb M, Mudad R: Correlating ISEND and Tumor Mutation Burden (TMB) with Clinical Outcomes of Advanced Non-Small Cell Lung Cancer (ANSCLC) Patients on Nivolumab. In: JOURNAL OF THORACIC ONCOLOGY: 2017: ELSEVIER SCIENCE INC STE 800, 230 PARK AVE, NEW YORK, NY 10169 USA; 2017: S2004-S2005.

63. Xiao D, Pan H, Li F, Wu K, Zhang X, He J: Analysis of ultra-deep targeted sequencing reveals mutation burden is associated with gender and clinical outcome in lung adenocarcinoma. Oncotarget 2016, 7(16):22857-22864.

Page $12 / 20$ 
64. Rizvi NA, Hellmann MD, Snyder A, Kvistborg P, Makarov V, Havel JJ, Lee W, Yuan J, Wong P, Ho TS et al: Cancer immunology. Mutational landscape determines sensitivity to PD-1 blockade in non-small cell lung cancer. Science (New York, NY) 2015, 348(6230):124-128.

\section{Figures}

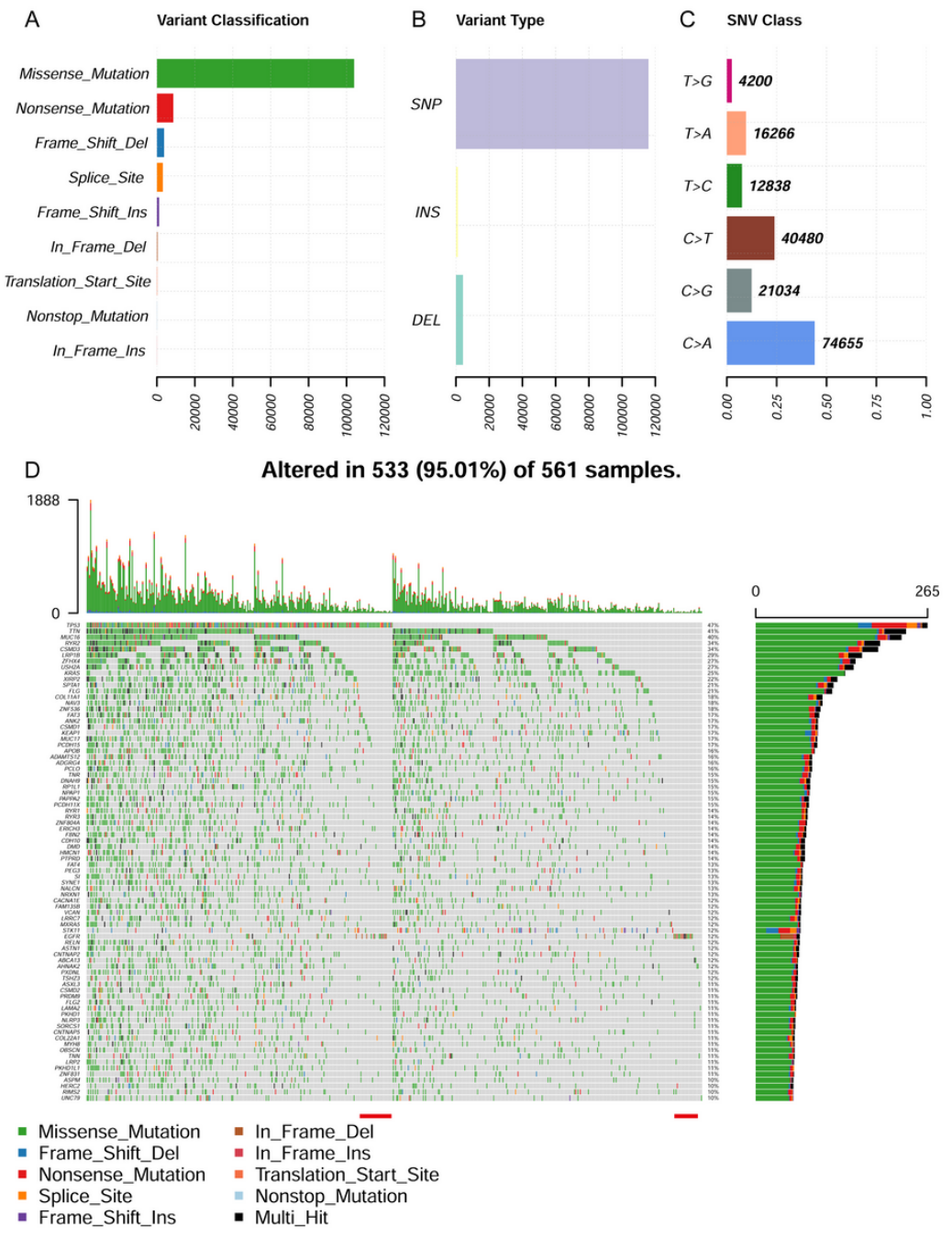

\section{Figure 1}

The landscape of mutation in LUAD samples. (A) Missense mutation was the most mutation type; (B) SNPs showed higher frequency than deletions or insertions; (C) C>A occupies the most common type of SNV; (D) Landscape of mutation profiles in LUAD samples. The top 80 genes with mutation probability in each sample (TMB $>0, N=533$ ) was shown in the waterfall plot, where various colors meant various mutations types. The bar plot above the waterfall plot exhibited the number of mutation burden. The red lines below the waterfall plot indicated the enriched EGFR mutant population. LUAD, lung adenocarcinoma; Del, deletion; Ins, insertion; SNP, single-nucleotide polymorphism; SNV, single-nucleotide variant; TMB, tumor mutational burden; N, number. 


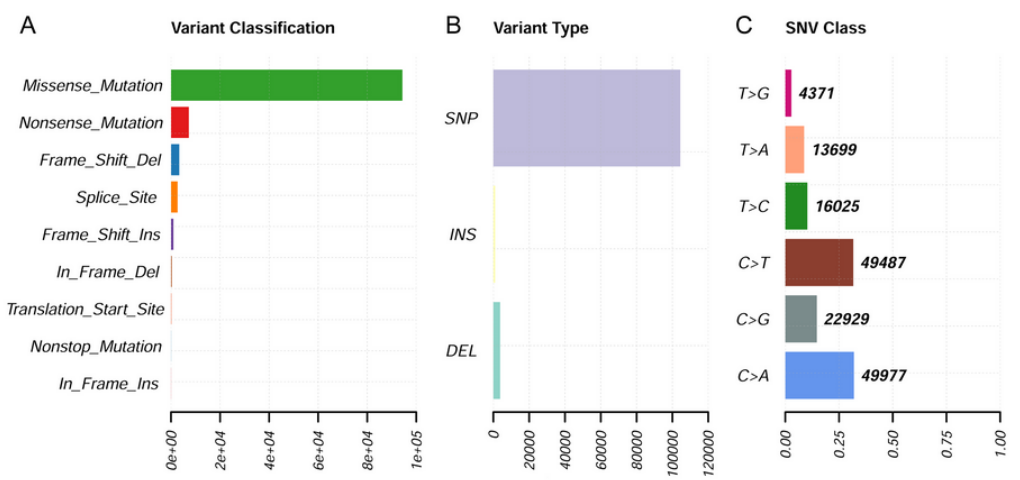

D

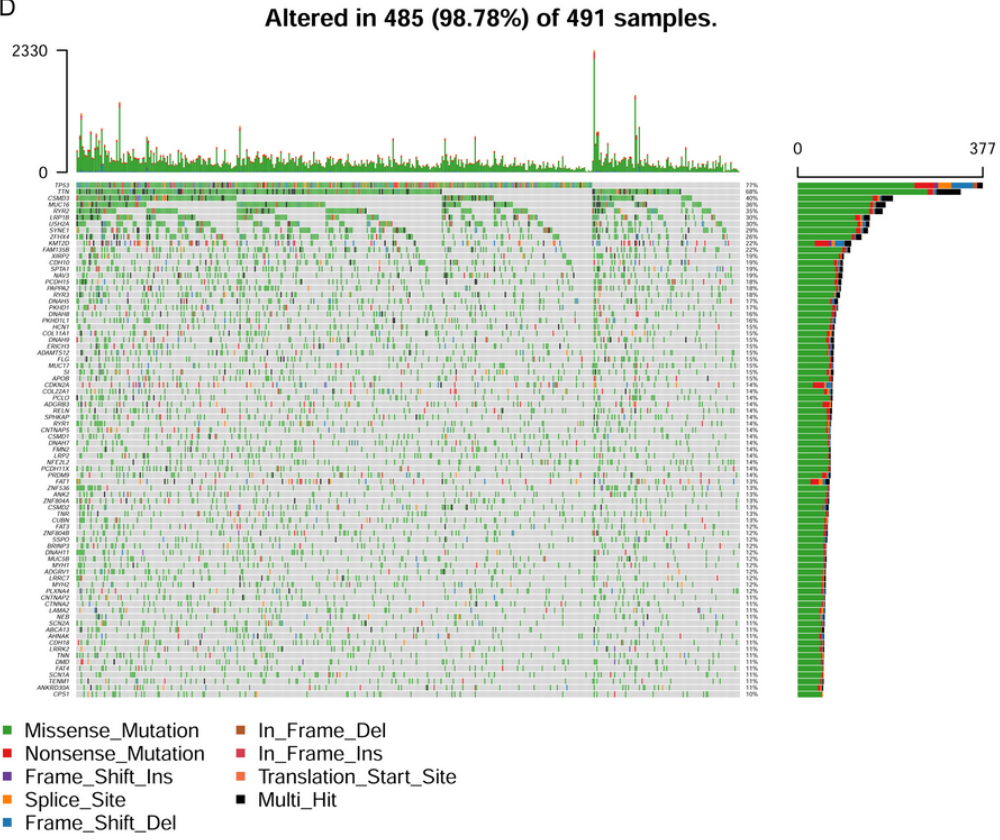

\section{Figure 2}

The landscape of mutation in LUSC samples. (A) Missense mutation was the most mutation type; (B) SNPs showed higher frequency than deletions or insertions; (C) C>A occupies the most common type of SNV; (D) Landscape of mutation profiles in LUSC samples. The top 80 genes with mutation probability in each sample (TMB > 0, N = 485) was shown in the waterfall plot, where various colors meant various mutations types. The bar plot above the waterfall plot exhibited the number of mutation burden. LUSC, lung squamous cell carcinoma; Del, deletion; Ins, insertion; SNP, single-nucleotide polymorphism; SNV, singlenucleotide variant; TMB, tumor mutational burden; N, number.

\section{A Variants per sample Median: 140}

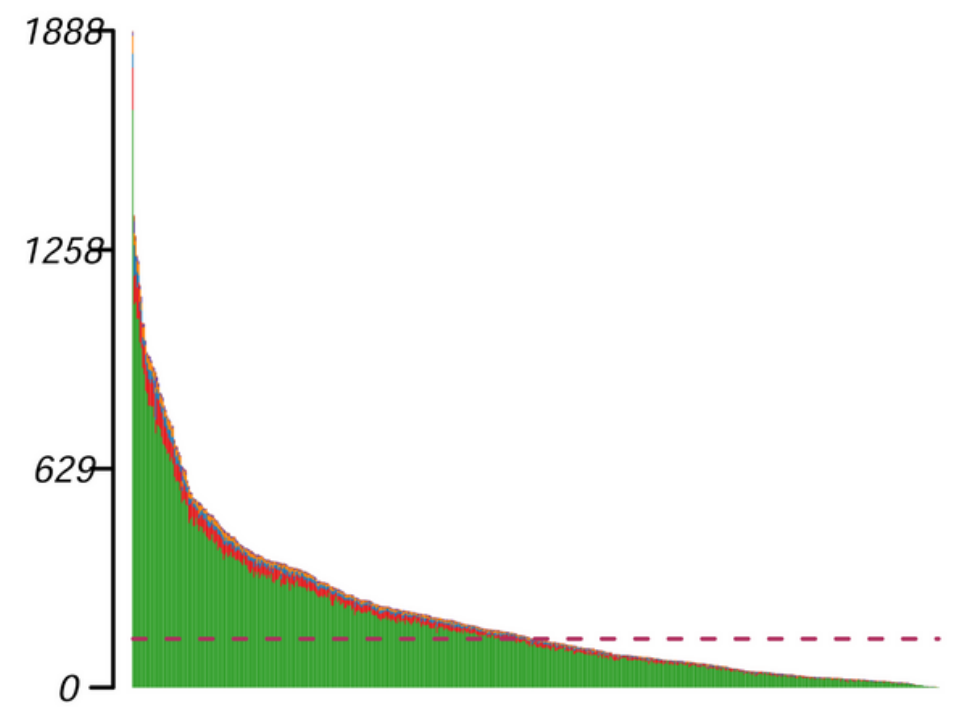

\section{B Variants per sample Median: 182}

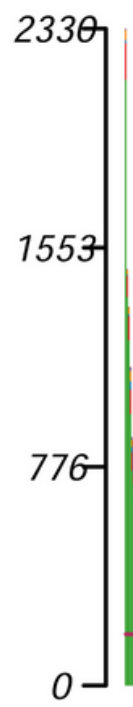


Figure 3

Mutation load value for each patient in LUAD (A) and LUSC (B).

A

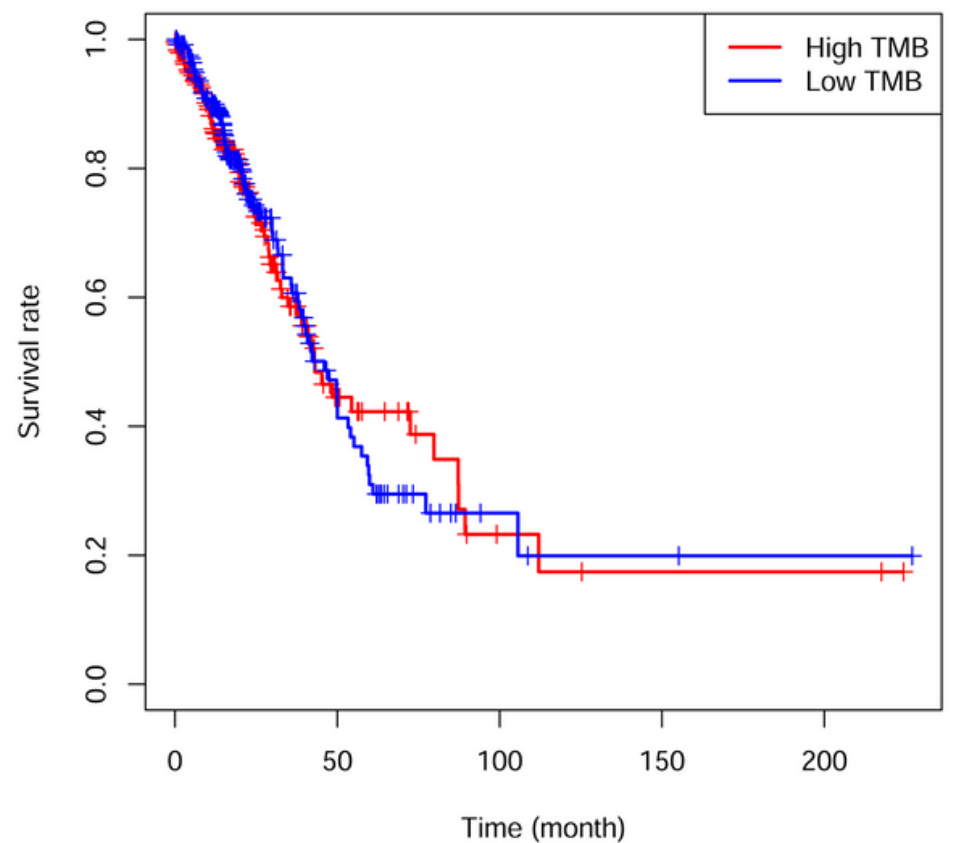

B

$\operatorname{TMB}(\mathrm{p}=0.602)$

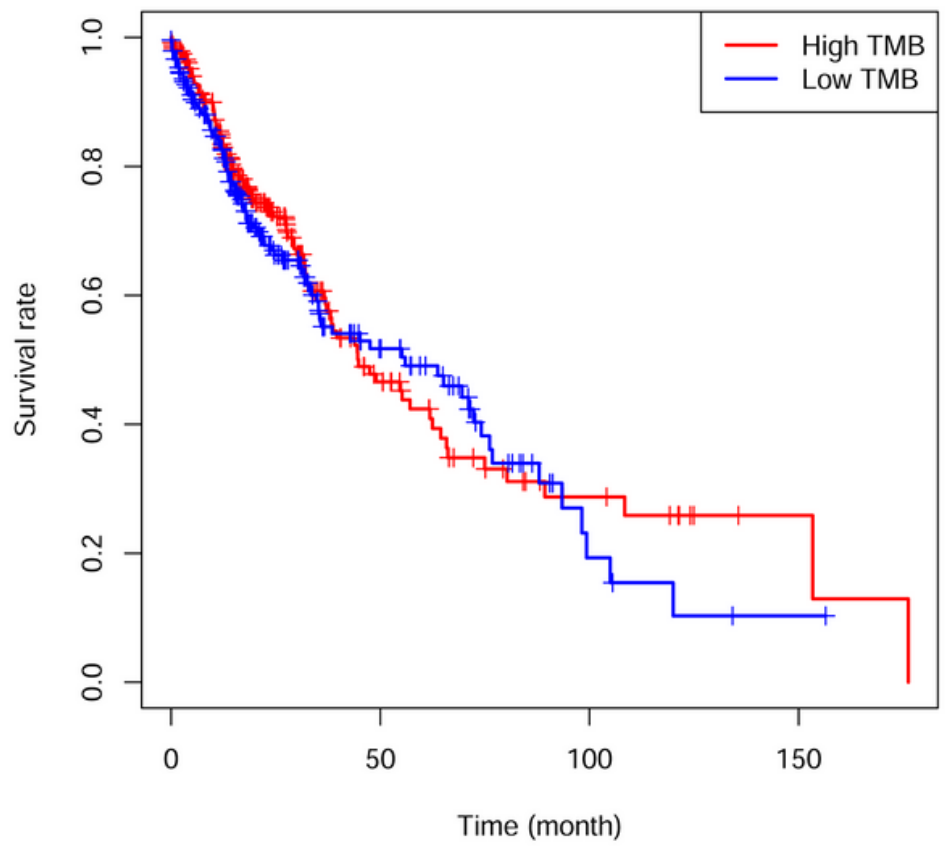

Figure 4

Kaplan-Meier survival curves with log-rank tests of high TMB and low TMB groups in LUAD (A) and LUSC (B).
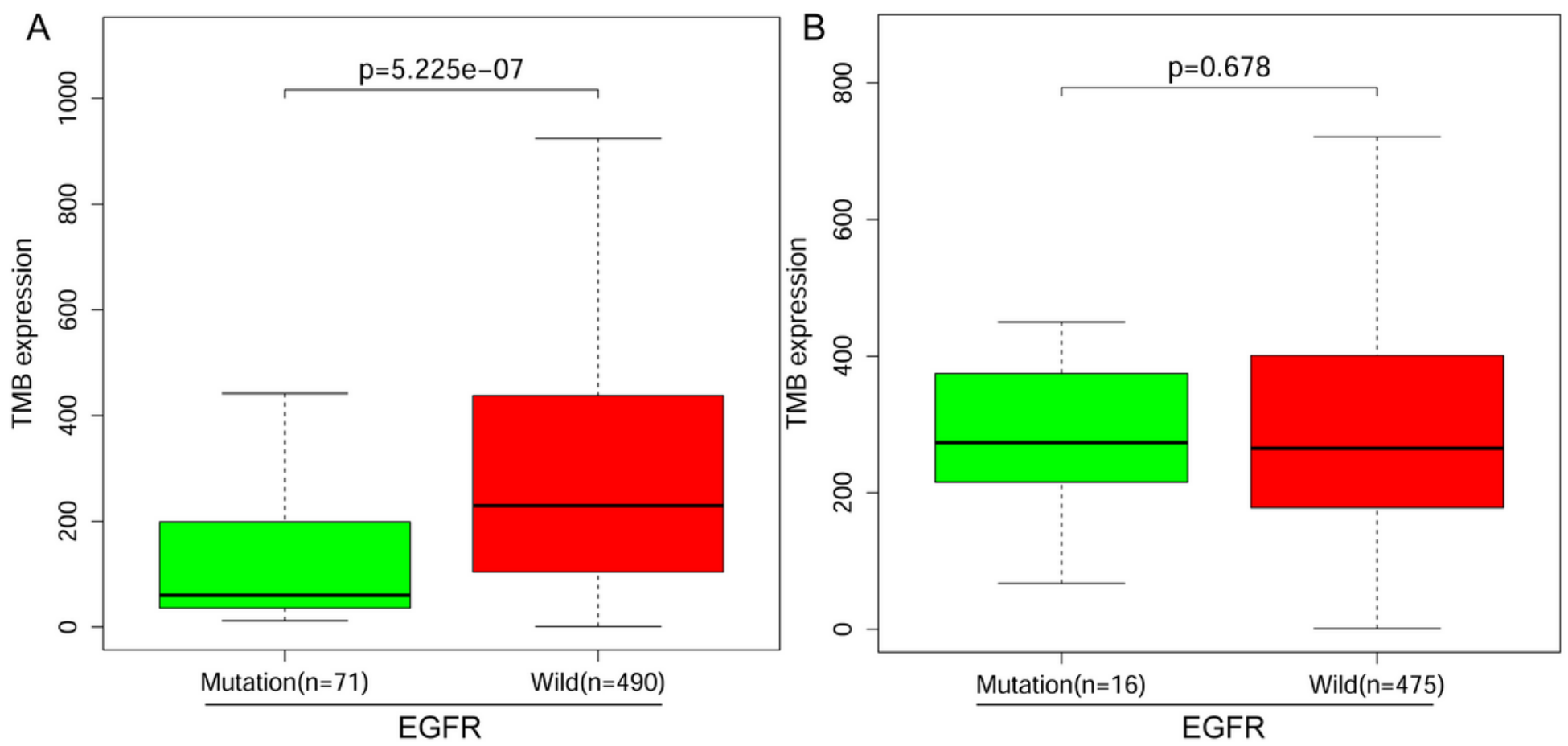

Figure 5

Relationship between EGFR mutation status and TMB value in LUAD (A) and LUSC (B). 

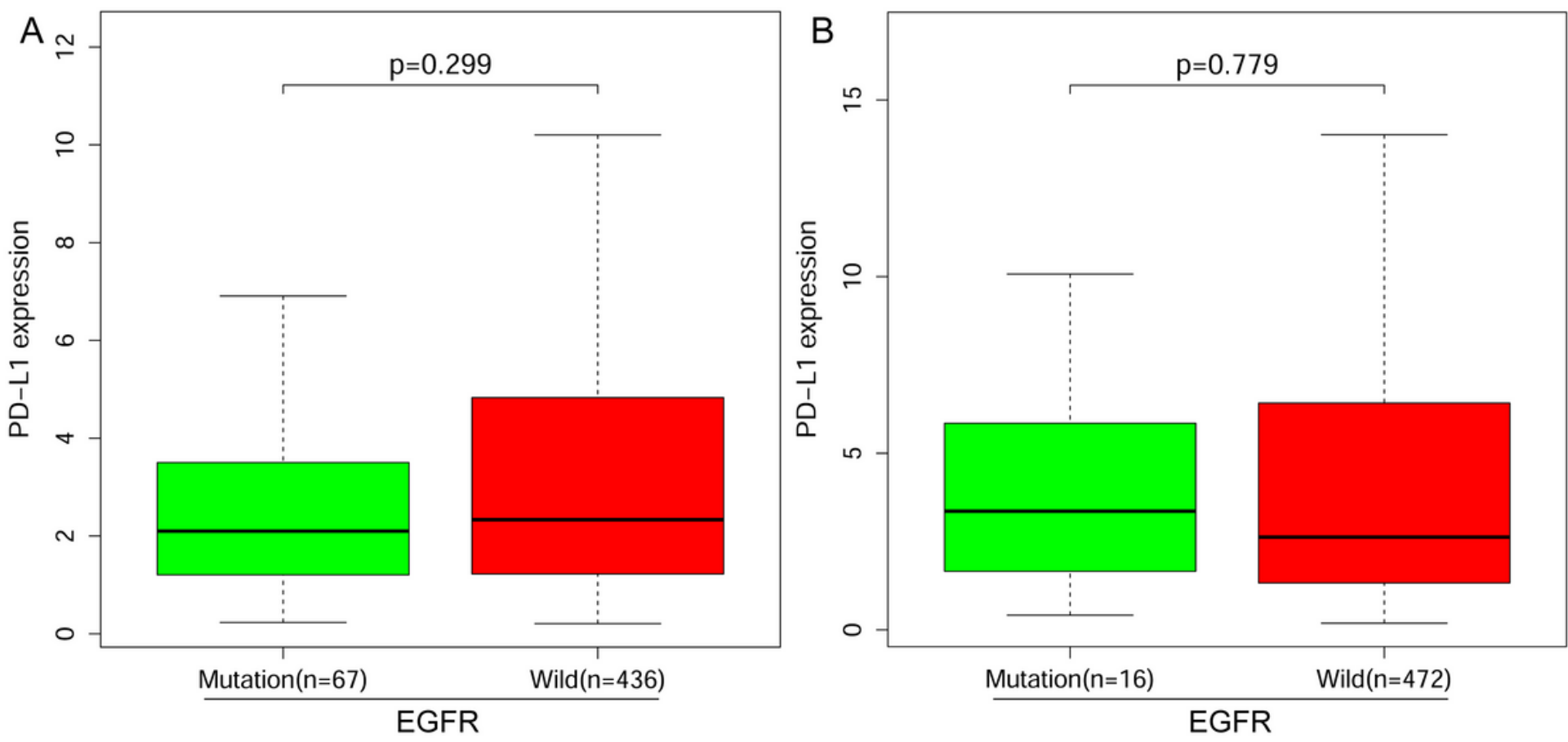

Figure 6

Relationship between EGFR mutation status and PD-L1 expression in LUAD (A) and LUSC (B).

Records identified through database searching $(n=25133)$
Additional records identified through other sources $(n=3)$

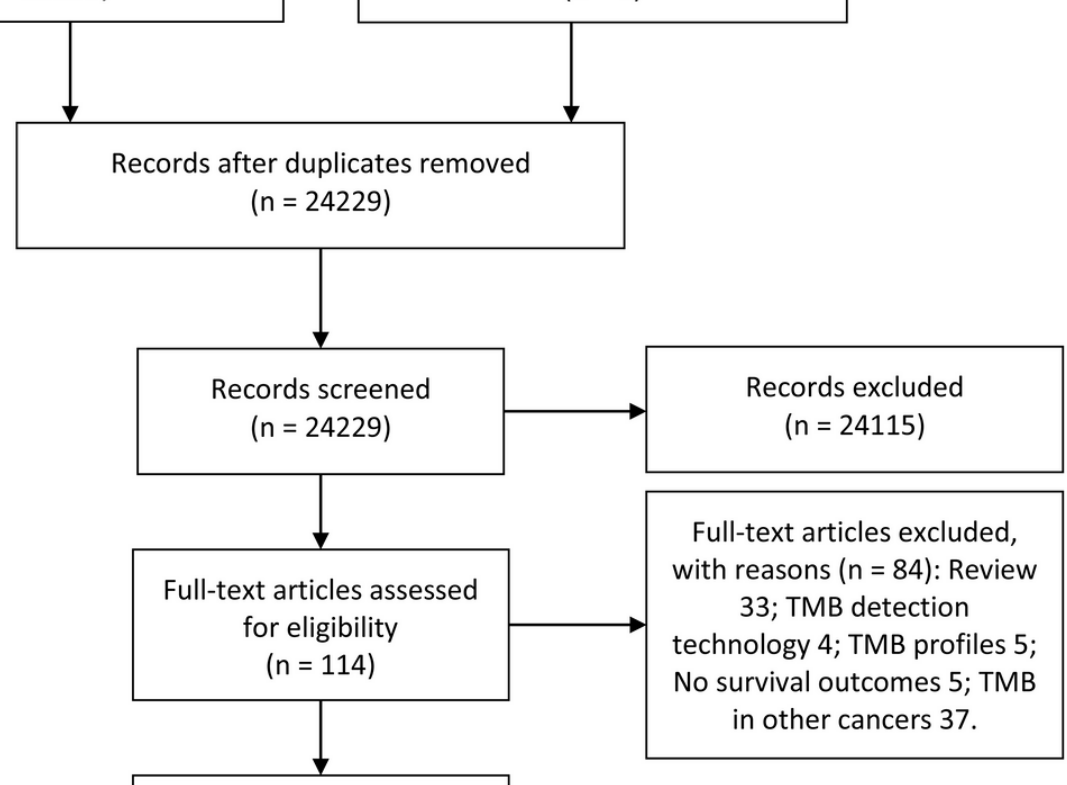

Studies included in qualitative synthesis $(n=30)$

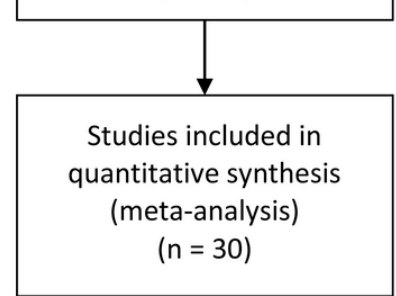

Figure 7 
Flow chart of searching the relevant studies included in this meta-analysis.

A

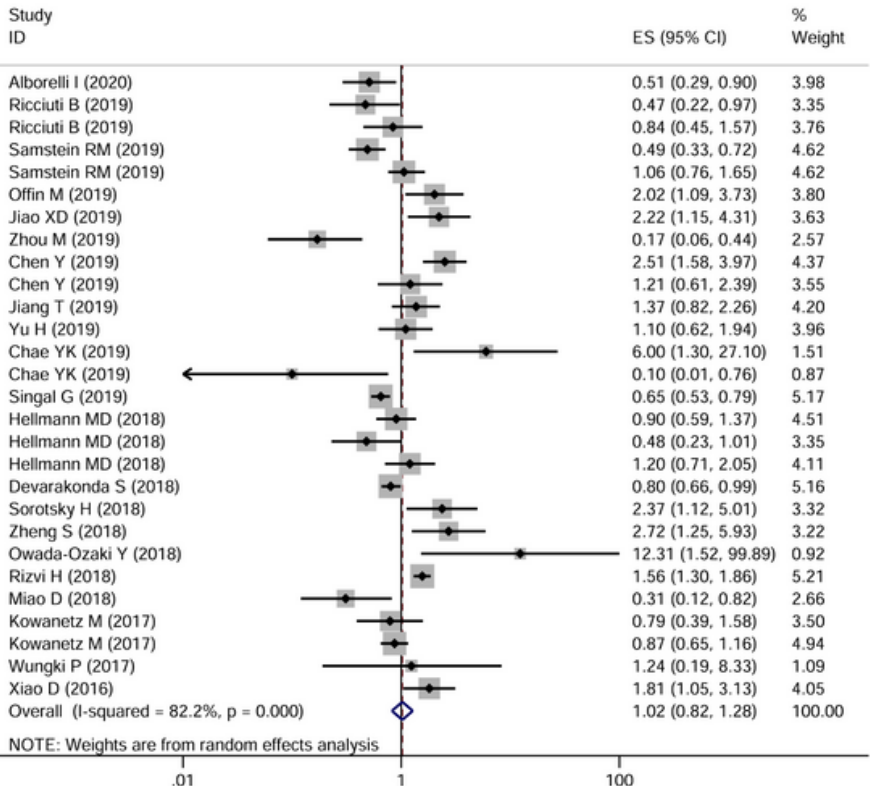

B

Study
ID

Figure 8

Meta-analyses of overall survival (A) and progression-free survival (B) of total lung cancer patients.

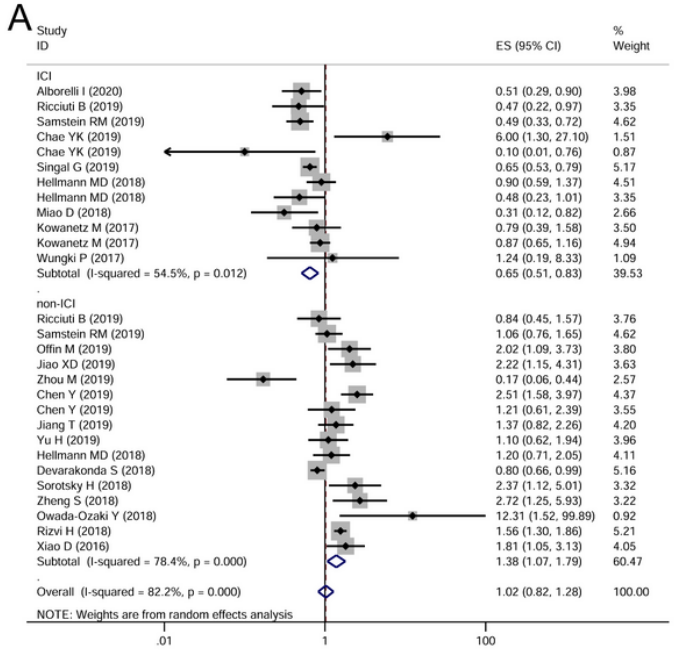

C

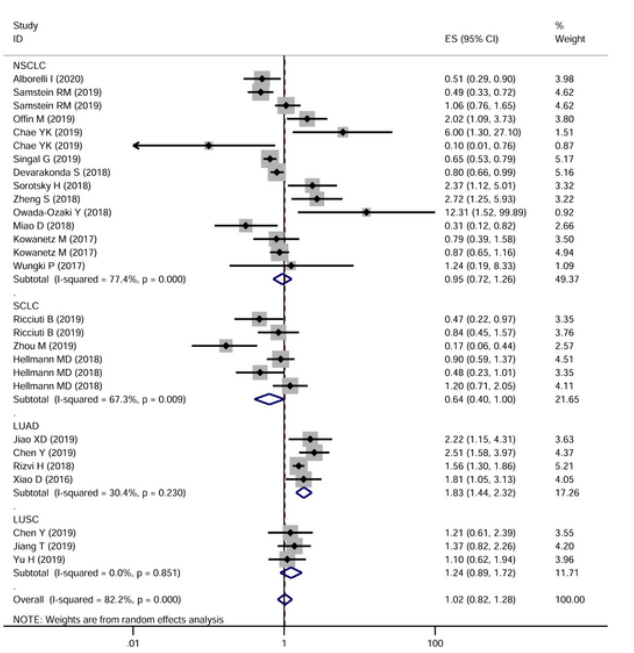

$\mathrm{B}_{\text {sut }}$

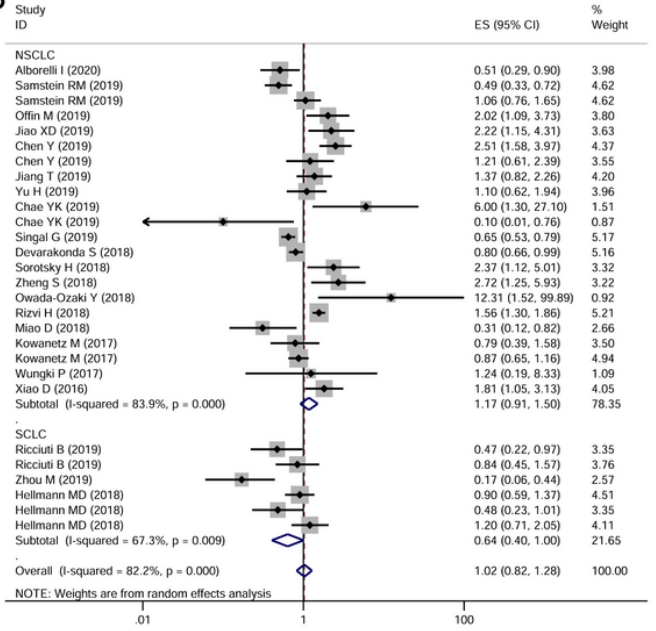

D

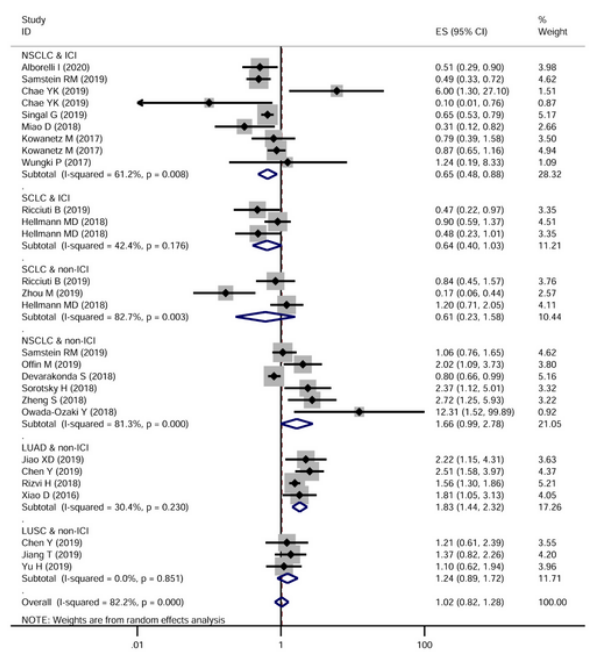

Figure 9 
Subgroup analyses of overall survival in lung cancer patients based on immunotherapy and pathological type. (A) Subgroup analysis based on immunotherapy. (B) Subgroup analysis based on NSCLC and SCLC. (C) Subgroup analysis based on NSCLC (separating LUAD and LUSC) and SCLC. (D) Subgroup analysis by combined pathological type and immunotherapy.
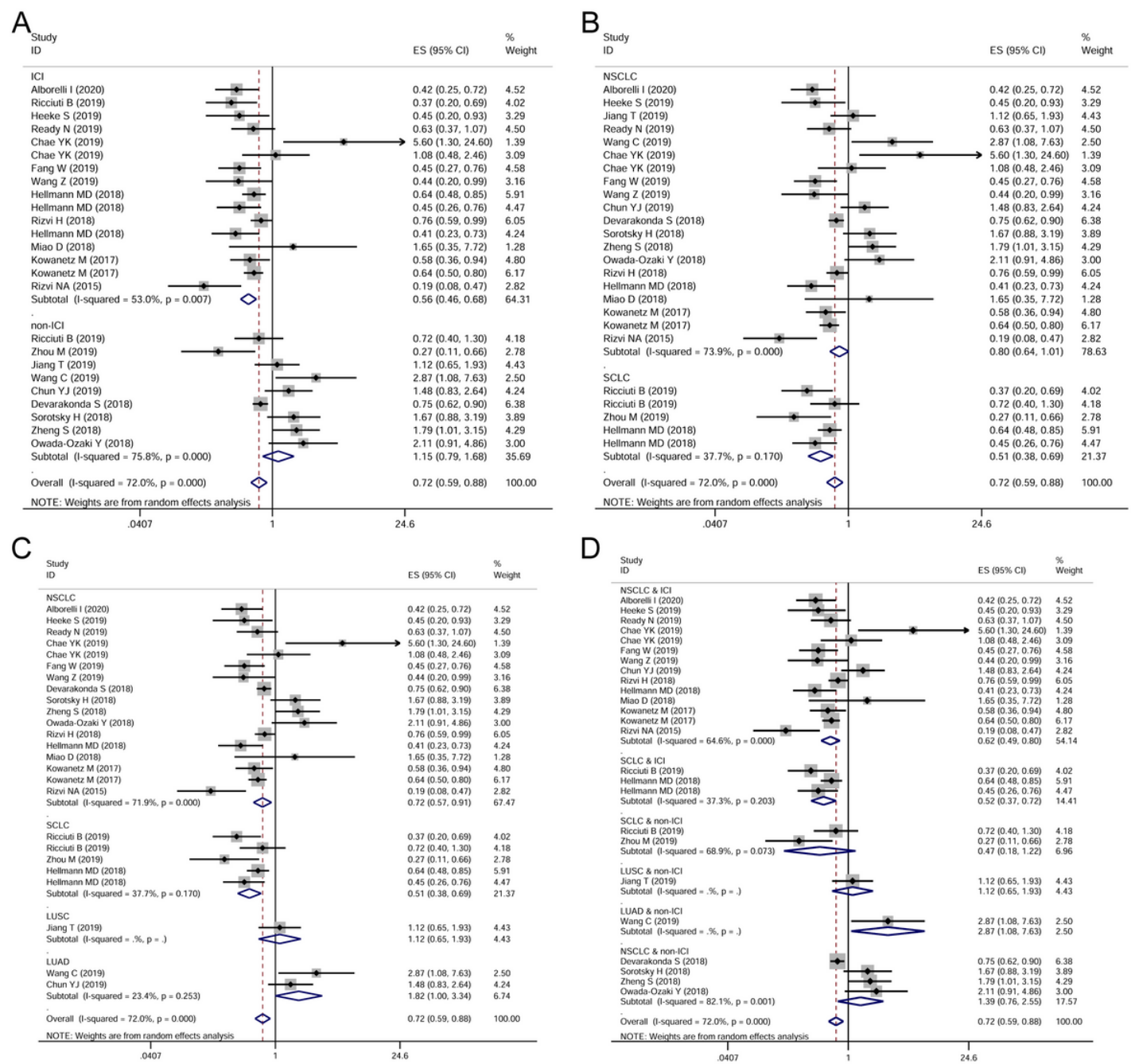

\section{Figure 10}

Subgroup analyses of progression-free survival in lung cancer patients based on immunotherapy and pathological type. (A) Subgroup analysis based on immunotherapy. (B) Subgroup analysis based on NSCLC and SCLC. (C) Subgroup analysis based on NSCLC (separating LUAD and LUSC) and SCLC. (D) Subgroup analysis by combined pathological type and immunotherapy. 

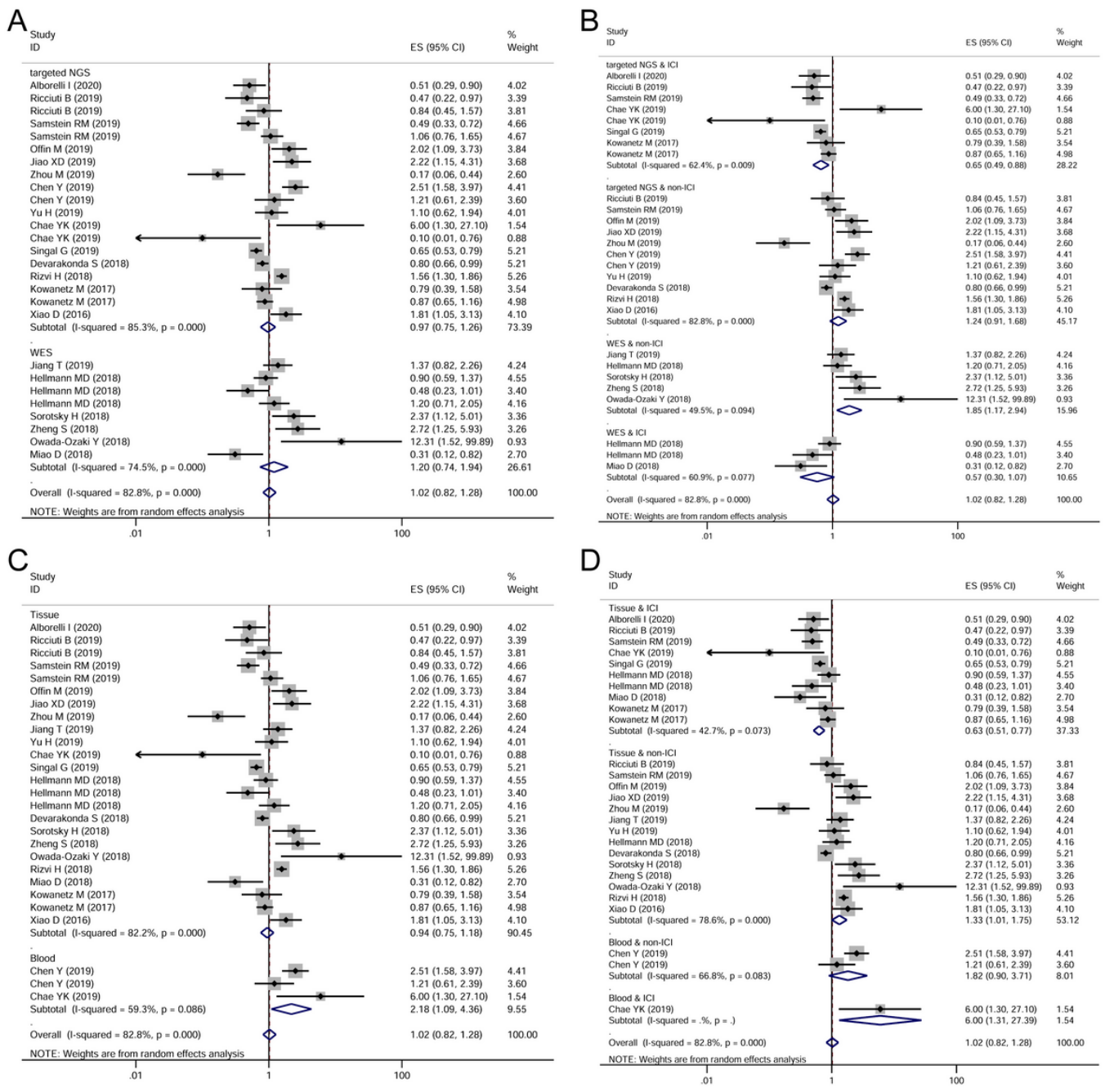

\section{Figure 11}

Subgroup analyses of overall survival in lung cancer patients based on test methods, sample sources, and immunotherapy. (A) Subgroup analysis based on test methods. (B) Subgroup analysis based on combined test methods and immunotherapy. (C) Subgroup analysis based on sample sources. (D) Subgroup analysis by combined sample sources and immunotherapy. 
A
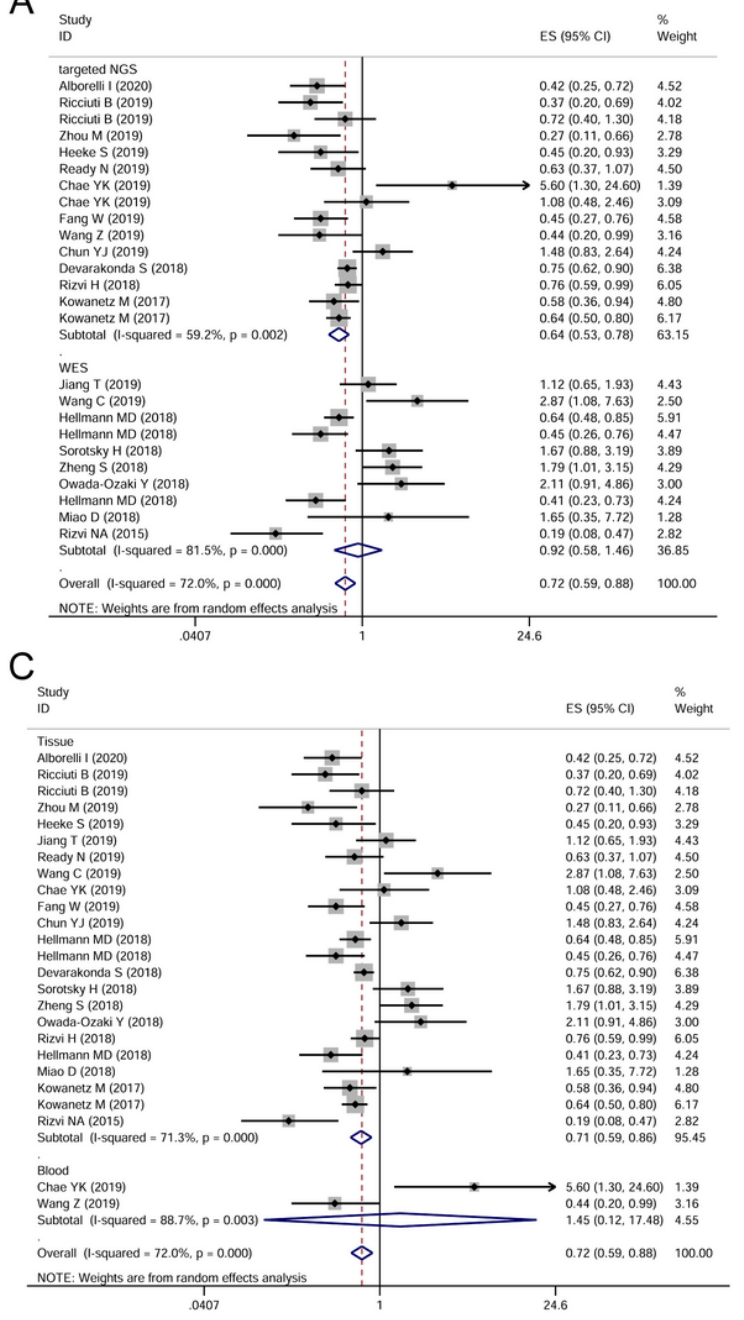

B

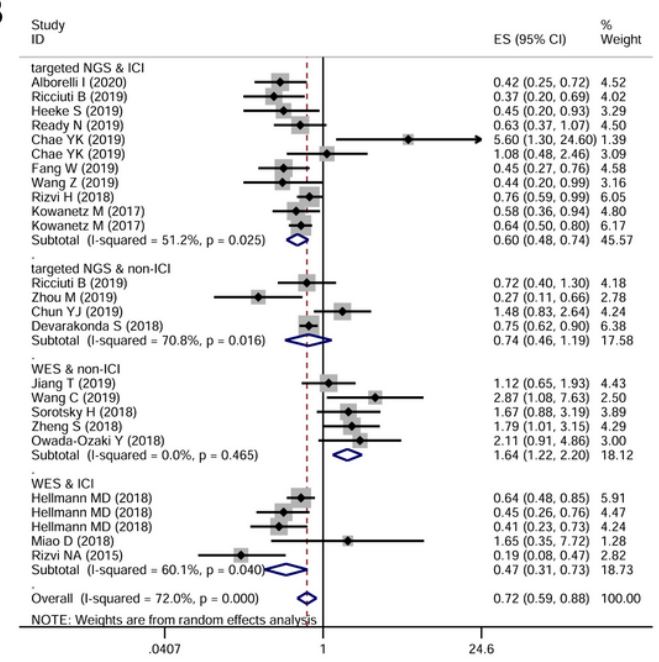

$\mathrm{D}_{\substack{\text { Sludy } \\ \text { ID }}}$

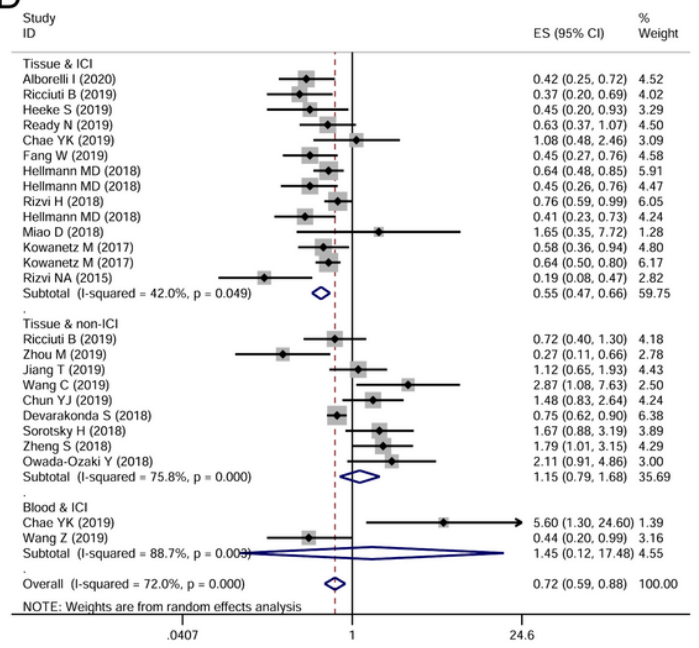

\section{Figure 12}

Subgroup analyses of progression-free survival in lung cancer patients based on test methods, sample sources, and immunotherapy. (A) Subgroup analysis based on test methods. (B) Subgroup analysis based on combined test methods and immunotherapy. (C) Subgroup analysis based on sample sources. (D) Subgroup analysis by combined sample sources and immunotherapy.

\section{Supplementary Files}

This is a list of supplementary files associated with this preprint. Click to download.

- PRISMAChecklist.doc 